\title{
HIV Populations Shift After Tuberculosis Associated Immune Reconstitution Inflammatory Syndrome: Implications For HIV Remission
}

Camille Lange ( $\nabla$ camille.lange@nih.gov )

National Institutes of Health

Maura Manion

National Institutes of Health

Natalie Lindo

National Institutes of Health

Robert Gorelick

National Institutes of Health

Ana Ortega-Villa

National Institutes of Health

Kristi Huik

National Institutes of Health

Christian Gonsalves

National Institutes of Health

Gregg Roby

National Institutes of Health

Virginia Sheikh

Food and Drug Administration

Catherine Cai

National Institutes of Health https://orcid.org/0000-0003-1855-7950

Luxin Pei

Nation Institute of Health

Elizabeth Laidlow

National Institutes of Health

Paula Rote

National Institutes of Health

Jennifer Bell

National Institutes of Health

Adam Rupert

Leidos (United States)

Joseph Adelsberger 
Leidos (United States)

\section{Wei Shao}

Leidos Biomedical Research, Inc., Frederick National Laboratory for Cancer Research

\section{Martha Nason}

$\mathrm{NIH}$ https://orcid.org/0000-0002-0110-881X

\section{Frank Maldarelli}

$\mathrm{NIH}$

\section{Irini Sereti}

National Institutes of Health https://orcid.org/0000-0002-0076-0224

\section{Article}

Keywords: Tuberculosis associated immune reconstitution inflammatory syndrome (TB-IRIS), combination antiretroviral therapy (cART)

Posted Date: November 30th, 2020

DOI: https://doi.org/10.21203/rs.3.rs-105550/v1

License: (1) (1) This work is licensed under a Creative Commons Attribution 4.0 International License. Read Full License 


\section{Abstract}

Tuberculosis associated immune reconstitution inflammatory syndrome (TB-IRIS) is a serious complication of starting combination antiretroviral therapy (CART). TB-IRIS emerges early after cART initiation and is characterized by rapid expansions of TB-specific CD4+ T cells and high levels of inflammatory mediators driven by CD4+ T cells. The effects of TB-IRIS on HIV populations are unknown, but could result in profound expansion and elimination of HIV infected cells via cellular activation and acute inflammation. We investigated immediate and long-term effects of TB-IRIS on HIV infected cells with and without TB-IRIS. We measured plasma HIV RNA, cell-associated HIV RNA and HIV DNA levels and compared genetic characteristics of HIV populations after prolonged CART. We found that TB-IRIS was associated with more diverse HIV DNA populations and HIV reservoirs after IRIS were distinct from pre-therapy populations, suggesting that TB-IRIS can shape the HIV reservoir with detrimental implications for HIV remission strategies.

\section{Introduction}

The global burden of HIV infection remains significant with 37 million known HIV infections worldwide and approximately 2 million new HIV infections per year (1). Combination antiretroviral therapy (cART) is effective at suppressing, but not curing HIV infection, and is now accessed by more than $50 \%$ of all people with HIV (PWH) (1). Many newly diagnosed PWH are late presenters with advanced HIV/AIDS and CD4+ T cell counts below 100 cells/ $\mu \mathrm{l}$, who are frequently co-infected with opportunistic infections such as tuberculosis (TB) and are at substantial risk for developing immune reconstitution inflammatory syndrome (IRIS), a severe and potentially lethal complication after initiating cART (2-6).

IRIS is an aberrant inflammatory response that occurs within the first few weeks of cART in a proportion of individuals with advanced HIV/AIDS and co-infections (e.g., mycobacteria, fungi) or certain neoplasms (e.g., lymphoma, Kaposi Sarcoma). TB is a frequent comorbidity and the most common cause of death in PWH (7). The development of IRIS in TB co-infected people can result in substantial morbidity and mortality, requiring urgent and, at times intensive medical therapy to treat worsening pulmonary, abdominal, or systemic complications of TB $(8,9)$. As CART continues to be rolled out worldwide to those with advanced HIV/AIDS, TB-IRIS is becoming a more common medical management issue. In addition to the acute complications, TB-IRIS is also likely to have long-term effects on expansion and persistence of HIV infected CD4+ lymphocytes during CART that may influence potential future scalable remission strategies $(10,11) \cdot(12)$.

TB-IRIS is characterized by soluble inflammatory markers as well as expansions of TB-specific CD4+ T cells (13-17). Oligoclonal expansion of TB-specific CD4+ T cells in people with IRIS prior to the initiation of CART have potential implications for the pathogenesis of TB-IRIS after CART initiation (18). Simultaneously, the HIV reservoirs that persist on CART are established within HIV infected CD4+ T cell populations that are present prior to cART initiation, and then reconstitute with effective CART (19). The effects of IRIS on HIV-infected cell populations are unknown; it is possible that the profound increases in 
TB-specifc CD4+ cells that occur during IRIS result in a comparable expansion of HIV infected lymphocytes and the HIV reservoir. Alternatively, the inflammatory burst accompanying IRIS may facilitate elimination of HIV infected cells, as it may drive reactivation of latent proviruses, further shaping the proviral landscape.

We hypothesized that TB-IRIS events have long-term effects on the composition and structure of HIV infected cell populations that persist during CART and long after IRIS events have resolved. We studied a cohort of people with advanced HIV/AIDS with TB-IRIS, TB without IRIS or no TB coinfection and no IRIS, and analyzed clinical, immunologic, and virologic characteristics prior to and during CART. Detailed phylogenetic and population genetics analyses of HIV populations revealed evidence of long-term effects of IRIS on HIV-infected cell populations.

\section{Results}

\section{Study Participant Characteristics}

To investigate the effects of IRIS on HIV populations, we identified three groups of HIV infected study participants for detailed investigations: (i) individuals with active TB with clinical signs and symptoms and microbiologic confirmation at CART initiation, who were diagnosed with TB-IRIS after starting CART, according to protocol specified criteria based on AIDS Clinical Trials Group Criteria (updated January 10, 2009) (20-22) (TBl; N=9). (ii) Individuals enrolled with active TB who did not develop IRIS (TBNI; N=9) and; (iii) individuals enrolled without TB who did not develop IRIS (NTBNI; $\mathrm{N}=10$ ). The demographic characteristics of these groups were comparable (Table S1); the median age of study participants (54\% female) was 38 years, with no significant differences in age among study groups $(P>0.05$; unpaired ttests). All study participants had chronic HIV infection and the majority (18 of 28 participants) were infected with subtype B, C, or CRF02_AG. Study participants had numerous comorbid complications, including Kaposi sarcoma, chronic hepatitis B, Cryptosporidium, herpes simplex virus, and recent Pneumocystis pneumonia (Table S1). Study participants with TB-IRIS experienced symptoms of IRIS within a median of 11 days (IQR 11, 14 days) of starting cART. They continued CART and most underwent additional corticosteroid therapy for variable periods until IRIS symptoms were resolved [median of 113 days (IQR 71, 336)]. Eight of nine individuals with TBI received corticosteroids, and 4 were on immunosuppressive therapy at the time of sampling shortly after CART initiation.

\section{CD4+ $T$ cell increases after CART initiation}

PWH typically experience an increase in CD4+ T cell numbers within the first 6 months of initiating CART (23), with slightly reduced rates of increase in TB coinfected people $(23,24)$. Prior to CART initiation, all study participants had $\mathrm{CD} 4+\mathrm{T}$ cell counts $<100 \mathrm{cells} / \mathrm{mm}^{3}$, indicating advanced disease (25). There was no difference in baseline CD4+ T cell levels in the blood among study groups (Table S1, average of 45 cells $/ \mathrm{mm}^{3} ; 6.5 \%$ of total lymphocytes; $p>0.05$ ). Within the first 5 weeks of cART, study participants with IRIS experienced a transient increase in CD4+ T cell levels (total cells and percent of total 
lymphocytes), followed by reductions in these cell numbers (Figure S1). Overall, there were no significant differences in the rate of increase in CD4+ T cells in the blood during the first 6 months of CART, consistent with recent observations (26), and total CD4+ T cell counts increased in the blood by an average of +3 cells per week during the first 26 weeks of cART ( $p>0.05$; linear mixed-effects model). For the next 1-2 years of cART, CD4+ T cell levels continued to rise by an average of +1 cell per week.

\section{Increased levels of soluble inflammatory and coagulation markers with IRIS}

Substantial immune activation is a hallmark of $\operatorname{IRIS}(25,27,28)$. We evaluated the plasma levels of 20 pro-inflammatory biomarkers prior to and following CART initiation in our cohort. Study participants with TBI had higher levels of D-dimer, MPO, IL-27, IFNy, IL-6, TNF-a, IP-10, hyaluronic acid, and soluble (s)CD14, both prior to cART initiation ( $<<0.05$; Kruskal-Wallis tests; Table S2) and during IRIS $(p<0.05$; KruskalWallis tests; Table S3), compared to those who did not develop IRIS. No significant elevations were detected in pre-CART levels of CRP, IL-2, IL-10, IL-8, soluble CD163 or hepcidin (Table S2). These data demonstrate that individuals with TB-IRIS had substantial systemic inflammation, as previously reported (26).

\section{Higher pre-therapy plasma HIV RNA levels in people who develop IRIS}

To investigate the effects of IRIS on HIV populations, we first quantified pre-therapy HIV RNA levels in plasma prior to cART. Study participants who developed IRIS after CART initiation had higher pre-therapy plasma HIV RNA levels compared to those who did not ( $\log _{10}$ median [Range] copies $\mathrm{RNA} / \mathrm{mL}$ plasma:

TBI 5.9 [4.9 - 6.6]; TBNI 5.1 [4 - 5.5]; NTBNI 4.7 [4.3-6.4]) (Figure 1a). Several cytokines (e.g. TNF-a and IL2) were also elevated in TBI participants (Tables $S 2$ and S3), which have been correlated with increased HIV RNA production in vitro (29-32). When we analyzed cytokine levels and HIV viremia prior to cART initiation, we found strong correlations between pre-therapy plasma HIV RNA levels and a number of soluble biomarkers, most of which were significantly elevated at baseline in the TBI group: TNF-a, IL-2, IL6, IL-10, CXCL-10, IL-27 and D-dimer ( $R=0.4-0.8 ; p<0.05$; Spearman correlations) (Figure S2).

After initiating CART, HIV RNA levels declined in plasma with typical multiphase decay kinetics in all study participants (33-36). Within 49 weeks of starting cART, all participants achieved plasma HIV RNA levels below the lower limit of detection (LOD) of commercially available assays ( $<50$ copies $/ \mathrm{mL}$ ) and remained below this LOD for the duration of the study period (Figure S3). After long-term cART, quantification of HIV viremia with sensitive single copy assay (37) (Table S4) revealed detectable levels in $80 \%$ of all participants, with no significant differences in levels of viremia among the study groups $(p=0.52$; Kruskal-Wallis tests). Therefore, although study participants with IRIS had higher pre-therapy viremia than those without IRIS, CART sufficiently inhibited viral replication below commercial LOD in all study participants within the first year of therapy.

\section{The size of HIV infected cell populations is comparable with or without IRIS}


We investigated the effects of TB-IRIS on the size and composition of HIV infected cell populations by quantifying the abundance of HIV infected cells in peripheral blood by measuring levels of cell-associated (CA) HIV DNA levels in the peripheral blood lymphocytes $(38,39)$. For this analysis, pre-cART HIV infected cells were available for a subset of study participants. Since CD4+ cell populations may shift significantly after CART initiation, especially during IRIS events $(12,40)$, we quantified the levels of HIV infected cells as the concentration of HIV infected cells in PBMC (copies of CA HIV DNA per mL blood) (Figure S4a), as well as in CD4 cell populations (copies of CA HIV DNA per million CD4+ T cells) (Figure S4b).

Prior to CART initiation, there were no significant differences in the proportion of HIV infected cells among study groups (CA HIV DNA in TBI versus TBNI and NTBNI groups: $p>0.05$; Kruskal-Wallis tests).

Comparable levels of HIV infected cells in the absence of cART indicate that the higher pre-therapy in HIV RNA levels in the TBI group could not be attributed to larger proportions of HIV infected cells.

After CART initiation, and as expected $(29,38,41,42)$, CA HIV DNA levels $(37,39)$ decreased in all evaluable study participants (Figure S5) as infected cells were eliminated. CA HIV DNA levels in the TBI study group remained comparable to the TBNI and NTBNI study groups after short-term cART and with prolonged cART (TBI vs TBNI and NTBNI; $p>0.05$, linear mixed-effects model). These data indicate that IRIS events neither expanded nor contracted the overall levels of HIV infected cells.

\section{No increases in cell-associated HIV RNA during or following IRIS}

Next, we investigated the effects of IRIS on CA HIV RNA production (37) in vivo, and quantified HIV RNA levels of total PBMC populations, normalized to the levels of HIV DNA present in the cells from which they were derived. Pre-therapy HIV RNA production (CA HIV RNA:DNA ratios) in cell derived material was substantial in all study participants tested regardless of study group (Figure 1b), and although pretherapy plasma viremia was higher in those with $\mathrm{TBI}$, there were no significant differences in pre-therapy CA HIV RNA production between individuals with TBI and TBNI. CA HIV RNA:DNA ratios decreased significantly after CART initiation in all study participants and there were no significant differences in HIV RNA production among study groups, during IRIS after short-term cART (Figure 1c), or after long-term cART (Figure 1d) ( $p>0.05$; Kruskal-Wallis tests). Analysis of this limited dataset demonstrated that, despite clinical and laboratory evidence of vigorous broad increases in pro-inflammatory mediators, those with IRIS did not have elevated levels of CA HIV RNA in PBMC compared to individuals without IRIS.

\section{TB-specific HIV infected CD4 cells are present, expand and persist after long-term cART}

TB-antigen (TB-Ag) driven polyclonal expansion of CD4+ T cells is an important mechanism implicated in IRIS pathogenesis $(14,43)$ and is reported to originate from cells that were present prior to cART initiation (18). We utilized a series of functional assays, where we stimulated CD4+ T cells derived from patient PBMC with TB-Ag (purified TB protein derivative; PPD) to determine if after long-term CART, these TB-Ag responsive CD4+ T cells included HIV infected cells, and if such cells were capable of HIV production in response to TB-Ag. We adapted the ultrasensitive HIV-Flow (44) approach to detect HIV infected CD4+ T cells obtained after prolonged CART, which produced virus in response to PPD exposure. 
PBMCs were available from 15 study participants (5 participants per study group) after long-term cART. We enriched for $C D 4+T$ cells via magnetic-bead depletion of CD8+, CD56+ and CD19+ cells. CD4+ T cells were cultured with IL-2 and PPD or antiCD3/antiCD28 beads (Dynabeads $\AA$ ) or a control with no activation agent. Growth media contained a nucleoside analogue and integrase inhibitor (200nM lamivudine and raltegravir respectively) to block new cycles of HIV infection during the stimulation. After 36 hours, cells were stained for production of p24 using a dual antibody staining strategy (28B7/KC57) (44).

In the absence of stimulation, 3 study participants with TB and 2 without TB had spontaneous HIV p24 production (Figure 1e). In the presence of PPD, the majority of the TB co-infected group (8/10 participants) exhibited HIV p24 production in 0.5-3.5 cells/million CD4+ T cells in response to PPD, which was greater than the TB-Ag response in the NTB group $(p<0.05)$. These results indicate that a proportion of HIV infected cells were TB-specific, activated by TB-Ag, and can produce virus. TB-specific HIV infected cells were present in individuals with and without IRIS and there were no statistically significant differences in concentrations of TB-specific HIV infected cells between those with and without IRIS (Median [95\% Cl Upper; Lower] $=1.2[1.2 ; 1.9]$ versus $0.5(1.0 ; 2.4)$ respectively; $p>0.1$ Kruskal-Wallis).

We further quantified the HIV populations in cells that were responsive to TB in 2 study participants with TBI (P6 and P7). PBMC obtained from P6 during IRIS (week 12 of cART), and from both individuals after long-term cART (week 96 and week 144 from P6 and P7 respectively) were incubated with PPD for 4 hours, sorted into CD4+ IFNg+/- subsets, and then further sorted on CD45RO+/-. HIV single copy assays were used to investigate levels of CA HIV DNA in CD4+CD45RO+ IFNg+ cells and substantial levels of HIV DNA were present in cells from both individuals; 8,300 copies HIV DNA per million TB-Ag responsive cells were present during IRIS in P6; and 6,300 and 8,700 copies HIV DNA per million TB-Ag responsive cells after long-term cART in P6 and P7 respectively). These results demonstrated that TB-Ag responsive CD4+ T cells obtained during IRIS and after long-term cART contained substantial numbers of HIV infected cells and that overall, TB-specific HIV infected cells make up a proportion of CD4+ T cell populations that were undergoing reconstitution during IRIS. These cells persist and remain responsive to TB-Ag after prolonged CART, long after TB resolution.

\section{TB-IRIS is associated with more diverse HIV and HIV infected cell populations}

The persistence of TB-specific HIV infected CD4+ T cells in co-infected people suggests that TB and IRIS have long-term effects on the structure of HIV infected populations. To investigate their effects on the composition and structure of HIV populations, we obtained single genome sequences from plasma derived HIV RNA and PBMC derived HIV DNA prior to and after the initiation of CART in 25 of the study participants. We used phylogenetic and population genetics approaches to define the structure of these populations, and we determined if HIV populations shifted after the introduction of cART. As our studies identified that TB-specific HIV infected cells were present throughout cART, we also analyzed the HIV populations present in the cells and media from the ex vivo TB-Ag stimulations of CD4+ T cells after longterm cART from P6 and P7. 
We first determined the genetic diversity of HIV populations by measuring average pairwise distance (APD) of single genome sequences (Table S5). No significant differences in the genetic diversity of plasma HIV RNA were detected in any of the study groups prior to initiating cART (Figure 2a; $p>0.05$ for all comparisons). Several of the participants in the TBI had relatively high genetic diversity in plasma HIV RNA (APD >1.9\%), which is higher than reported in a number of previous studies (45-48). The source of this increased genetic diversity is uncertain and further analysis revealed no correlation between plasma HIV RNA levels and APD ( $p>0.05$; Spearman correlation), however there was a strong positive correlation between IL-2 levels and APD of pre-therapy plasma HIV RNA populations (Figure $2 b)(R=0.73, p=0.03$; Spearman correlation), supporting the possibility of antigen driven proliferation and expansion of the cellular viral reservoir in those with TBI, which may be driving the genetic diversity of HIV populations.

Although the diversity of pre-therapy plasma HIV RNA populations was comparable among all study groups, there were minor differences in PBMC derived HIV DNA populations. Pre-therapy APD of PBMC HIV DNA populations in participants with TBI was modestly higher than corresponding levels in NTBNI groups (Figure 2c, Table S5: APD 1.8\% versus 1.3\% respectively; $p=0.04$; Kruskal-Wallis tests). Study participants with TBI also had more diverse HIV DNA populations during IRIS compared to the diversity of HIV DNA populations after short-term cART in non-IRIS groups (Figure 2d, APD 1.8\% versus $1.2 \%$ respectively; $p=0.03$ ). After prolonged $c A R T$, however, there were no significant differences in genetic diversity of post-IRIS HIV DNA populations compared to those who had no prior IRIS event or pre-existing TB (APD 1.9\% versus 1.3\% respectively; $p>0.05$; Figure $2 e$ ). A temporal trend analysis of genetic diversity in study participants who developed symptoms of IRIS revealed a greater overall diversity in HIV DNA populations on cART compared to those who did not experience IRIS (Figure 2f; $p=0.01$; linear mixedeffects), demonstrating a greater overall genetic diversity in TBI study participants.

\section{Evidence of potential clonal expansions of HIV infected cells during IRIS}

To further investigate the structure of HIV populations we performed detailed phylogenetic analysis of single genome sequences. In general, sequences from TBI, TBNI and NTBNI groups were highly diverse; however, in each group there were examples of identical sequences, suggesting they were potential clones. Additional clonal prediction scores (49) (Figure S6) confirmed these identical sequences had high probability of being members of cell clones. Phylogenetic analysis of single genome HIV sequences derived from CA HIV DNA populations revealed that portions of these populations comprised of identical sequences, which may represent clonally expanded HIV infected cell populations. Potential HIV clones (49) were comparable among study groups; median (range) frequencies of HIV clones in all study groups combined was $5 \%(0$ - 38) prior to CART initiation, $13 \%(0-43)$ shortly after CART initiation and $18.5 \%(0$ 59 ) after long-term cART ( $p>0.05$ among all study groups; Kruskal-Wallis tests). We did observe an increase in the frequency of identical HIV DNA sequences in participants with TBI after short-term cART (pre-therapy median [range] $=0 \%[0$ - 19] versus short-term cART 21\% [0 - 43]; $p=0.03$; One-way Anova test) (Figure 3a), but not in those who did not experience IRIS (Figure 3b and 3c). 
After prolonged cART, identical sequences remained detectable in all study groups, with no further increases in the TBI group after IRIS. We did not observe a relationship between the proportion of identical HIV DNA sequences in the TBI group with continuous CART $(p=0.9$; Poisson regression analyses), but in the absence of IRIS, there was an increase in the proportion of identical sequences detected on cART ( $p=0.0003$ in the TBNI group and $p=0.02$ in the NTBNI group).

This phylogenetic analysis of HIV populations suggested fundamental differences in the structure of CD4 populations of the TBI group. To characterize the structure of the entire CD4 population, we determined the distribution $T$ cell receptor rearrangements using high throughput sequencing, from which we investigated the diversity of the populations using Shannon entropy analyses. Figure $3 \mathrm{~d}$ illustrated the relatively low Shannon index of the TBI group, indicating a highly diverse population, while the Shannon index for TBNI was significantly higher ( $p=0.04$; Kruskal-Wallis test). These findings indicate that in the absence of previous IRIS, CD4+ T cell diversity in HIV/TB co-infected individuals is less diverse after longterm cART, CD4+ T cell expansions associated with IRIS include HIV infected cells and imply a more polyclonal population of expanded HIV infected cells with IRIS.

\section{Long-term shifts in HIV populations after IRIS}

Overall, phylogenetic analyses revealed that pre-therapy plasma and PBMC derived HIV populations were highly related; single genome sequences derived from plasma and PBMC appeared to be intermingled, with plasma and PBMC-derived sequences sharing large branches on the phylogenetic tree, and few distinct branches having bootstrap supports $>70$ (Figure S7). To rigorously investigate whether the HIV populations in PBMC and plasma were distinct, we performed detailed analyses for population subdivision and migration.

Population subdivision analysis (46) revealed pre-therapy HIV populations in plasma RNA and PBMC DNA as well-mixed, and derived from the same viral population ("panmictic populations"; Table S6). The presence of identical sequences may bias panmixia analyses, therefore we repeated these analyses with individual groups of identical sequences collapsed to single lineages; these repeat analyses confirmed that the pre-therapy populations were panmictic. An independent analyses for population shifts using migration analysis (Slatkin Maddison (50)) also determined that pre-therapy sequences in plasma and PBMC were intermingled, with no evidence of compartmentalization or non-random migration of variants (Table S7). Taken together, these data demonstrate that HIV populations in plasma and PBMC prior to cART initiation are highly diverse and well mixed.

To investigate the fate of these pre-therapy viral populations after CART initiation, we repeated these population genetics analyses using single genome sequences obtained from PBMC after short-term CART. After initiating CART, HIV populations continued to be genetically diverse. However, detailed fine structure analysis using population subdivision analyses revealed that population shifts took place in some participants. As illustrated in Figure 4, HIV DNA populations from 1/9 TBI participants (Figure 4a) and $3 / 16$ without IRIS (Figure $4 b$ and $4 c$ ) were shifted and were no longer panmictic with pre-therapy plasma HIV RNA populations after short-term cART. These data suggest that shifts in HIV-infected cell 
populations occur in some individuals early after CART initiation, but the presence of these shifts was not more frequent with IRIS.

We also analyzed PBMC DNA derived HIV populations after prolonged CART to determine if IRIS affected long-term persistence of HIV-infected cell populations. As shown in Figure 4, HIV populations after longterm cART remained highly genetically diverse, however, population shifts were significantly more common after long-term cART in individuals who experienced IRIS after starting therapy (6/9 participants with TBI) compared to those who did not experience IRIS (2/16 NI participants; $p=0.03$; Fisher-Exact test). In addition, all study participants with TBI had evidence of compartmentalization of HIV DNA populations (Slatkin-Maddison, probability of compartmentalization $p=0.002$ ) while only $3 / 16$ individuals without IRIS had evidence of compartmentalization. The differences in the frequency of compartmentalization in individuals with prior IRIS events was highly significant ( $p=0.0001$, Fisher's exact test). Taken together, these data indicate that TB-IRIS events fundamentally shift the long-term composition of HIV populations persisting during CART.

One study participant in the TBI group, P13, discontinued cART due to nonadherence and experienced a rebound in viral replication (186,000 copies HIV RNA/mL plasma) after 76 weeks of suppression of viral replication with CART. We analyzed the genetic characteristics of the viremia to determine if the rebound virus was distinct from pretherapy virus, as predicted by the panmixia and compartmentalization analyses. As shown in Figure S8, the predominant virus producing lineage was not clustered with the majority of proviral lineages detected during IRIS or after 64 weeks of cART. Panmixia analyses revealed the rebounded plasma viral population was distinct from pretherapy (Table S8), demonstrating that the HIV reservoir was distinct from pretherapy viremia. Of note, the rebound viremia was also distinct from the HIV DNA population at week 64 (Table S8), suggesting rebound viremia emerged from a highly diverse HIV DNA population.

These analyses indicate profound shifts in HIV populations after IRIS; the lineages of HIV infected cells persisting after IRIS, do not reflect the viral populations present in plasma at the time cART was introduced. Furthermore, these data suggest that the majority of infected cells that were producing HIV virions at CART initiation did not persist in the PBMC compartment after TB-IRIS. Furthermore, rebound viremia in P13 did not originate from the full spectrum of viral lineages replicating prior to CART initiation, but rather from a limited proportion of the proviral lineages persisting on CART. This finding suggests that in this individual, defective proviral lineages dominated the HIV infected cell populations that persisted on CART and not virus producing lineage largely responsible for rebound viremia.

\section{TB antigen-driven expansion of HIV infected cells as a mechanism of HIV persistence}

We showed that during IRIS and after long-term cART, some TB-Ag responsive cells from those with IRIS harbor HIV proviruses. Next, we investigated the possibility of clonal expansion of HIV infected cells of this phenotype as a possible mechanism of HIV persistence in these individuals, and if these cells were capable of producing HIV virions after long-term cART. The cellular yield from the ex vivo stimulation studies was insufficient to do detailed integration site analyses, therefore populations were analyzed 
using single genome sequencing and phylogenetic analyses. During IRIS, we obtained 20 sequences from CD4+CD45RO+IFNg+ cells derived from P6. After long-term CART we obtained 36 sequences from P6 and 26 sequences from $\mathrm{P7}$ from cells of the same phenotype. Several variants in both study participants were detected multiple times and first detected during IRIS.

In P6, there were 3 viral variants detected in HIV DNA populations during IRIS, with evidence of potential persistence via clonal expansion in response to TB antigen (Figure 5a). Two of these variants (Figure 5a, Variants 1 and 3 ) were first detected in $C D 4+C D 45 R O+I F N g+T$ cell populations responsive to $T B$ antigen during IRIS. Identical sequences were detected after 96 weeks of cART in this patient in HIV DNA populations from the same TB responsive cell phenotype, suggesting that these cells were present during IRIS and persisted for prolonged periods after IRIS subsided and TB infection resolved. Variant 3 was also detected in the HIV RNA derived from the supernatant of the cell culture after PPD stimulation, indicating that the cell of origin activated by TB-antigen stimulation was capable of producing HIV virions.

In P7, there were 2 viral variants detected during IRIS in PBMC HIV DNA populations with identical sequences detected after 144 weeks of CART in CD4+CD45RO+IFNg+ cells (Figure 5b). Variant 1 was detected in PBMC obtained prior to CART initiation, demonstrating potential persistence of these variants from pre-therapy lineages. HIV DNA populations within TB-Ag responsive cells were panmictic with pretherapy HIV RNA populations.

These findings demonstrate that TB-specific cells can harbor HIV proviruses and produce HIV virions within the context of coinfection and active TB disease prior to CART initiation. We found that HIV infected TB-specific CD4+ T cells with memory phenotypes can produce virions. Furthermore, both TB coinfection and IRIS can shape the HIV infected cell populations that persist on CART, potentially through mechanisms like clonal expansion. Finally, these proviruses can produce virions, inducible by TB antigens after years of CART, highlighting their clinical relevance and importance in considerations for viral remission strategies.

\section{Discussion}

IRIS is a severe inflammatory complication that develops in HIV infected people starting CART with advanced HIV/AIDS and comorbid infections, such as TB. TB-IRIS is a growing challenge as CART availability and accessibility are scaling-up in resource limited countries where advanced HIV and TB disease are more frequent $(1,7)$. As IRIS is characterized by rapid and vigorous CD4 responses and a surge of inflammatory cytokines, understanding the effects of IRIS on HIV infected T cell populations will also shed light on the forces shaping HIV reservoirs and our understanding of how these reservoirs may be eradicated.

Here we characterized HIV populations prior to and following the introduction of CART in people with advanced HIV/AIDS enrolled in prospective studies of IRIS $(21,22)$. Increased plasma HIV RNA levels have been previously reported as a risk factor for the development of IRIS upon initiating CART in TB coinfected people $(3,51)$, but the mechanism for these observations remains unknown. We confirmed 
elevated levels of HIV viremia and analyzed virologic parameters prior to initiating CART to determine if a larger reservoir of HIV infected cells, or exuberant immune activation was responsible for driving increased levels of virus production. We found no significant differences in the proportion of HIV infected cells present with or without IRIS, suggesting that the overall viral reservoir size was comparable across study groups. However, viremia did correlate with levels of soluble inflammatory biomarkers, indicating that immune activation, and not reservoir size is responsible for increased levels of viremia in those who subsequently develop IRIS on cART.

Shortly after CART initiation, the amount of HIV infected cells closely followed total CD4+ T cell counts and those who developed IRIS had significant increases in CD4+ T cell numbers, possibly reflecting the expansion of cells responding to TB $(18,40,43)$. HIV infected cells were present in IRIS associated CD4+ T cell expansions and we detected HIV infected cells that were specifically responding to TB-Ag in in vitro assays. In our phylogenetic analyses, we did not detect large groups of identical sequences that would have been suggestive of clonal expansion of a limited number of infected cells. Rather, the HIV proviruses, including those in TB responsive cells were genetically diverse, demonstrating many different proviruses within HIV infected, TB-specific cell populations.

We also found TB responsive cells persisting years after CART initiation, indicating their capacity for longlived persistence. Long-lived, TB responsive, HIV infected cells were identified through p24 staining, indicating that they were capable of producing structural HIV proteins, however additional studies are needed to determine whether TB-responsive, HIV infected cells produce replication competent virus. In addition, we assume that a larger proportion of CD4+ T cells responded to TB-Ag than we observed because HIV Flow is limited to p24 production, thereby underestimating the proportion of HIV infected cells that responded to TB-Ag. However, we found no differences in the levels of TB responsive cells with or without IRIS, demonstrating that such cells are not restricted to people with IRIS, but rather, they are a general characteristic of TB disease and clonally expand in response to TB-Ag. The presence of these cells long after TB has resolved suggests their persistence may be maintained by clonal proliferation.

IRIS resulted in profound shifts in the composition of the HIV infected cell reservoirs that persisted during CART. As shown in our model (Figure 6), in the absence of IRIS, the genetic composition of populations of HIV infected cells present in blood remained relatively stable on CART. In most study participants, the HIV infected cell populations sampled after several years of cART were panmictic with pre-therapy plasma HIV RNA populations. In contrast, population shifts occurred in individuals undergoing IRIS, such that for most individuals, the HIV infected cells responsible for pre-therapy virus population were not represented in the cell populations detected after long-term cART. We observed multiple migrations of new HIV infected cells into the long-term PBMC compartments in those who experienced IRIS, suggesting an influx of infected cells from outside the blood compartment was responsible for these population shift. It is possible that virus infected cells that were present in tissues (and not in PBMC) at the start of therapy gradually trafficked to PBMC. Such shifts predict that pretherapy virus population will not be the dominant source of replication competent HIV population. Overall, these findings demonstrate a profound and long-term effect of IRIS on the HIV proviral landscape.

Page 12/30 
We propose that the antigen specific immune response, as well as inflammation, are important drivers of clonal expansion and persistence in the HIV reservoir. As HIV infected, TB-specific cells were detected long after TB resolution, it is likely that homeostatic proliferation also has a role in maintaining these cells. Prior to CART initiation, HIV and non-HIV specific CD4+ T cells are proliferating and expanding within the context of chronic HIV disease. Active TB disease influences the HIV infected cell populations so that some of these HIV infected cells can also persist via clonal expansion after CART initiation. Therefore, these cells reconstitute as a result of TB-Ag driven cell proliferation and/or the mechanism of immune recovery as a result of CART. These diverse populations of HIV infected cells accumulate during IRIS and persist with long-term cART when both TB disease and IRIS have long resolved. IRIS adds yet another mode of HIV infected cell expansion through the pro-inflammatory response, thereby facilitating a reconstituted polyclonal CD4+ T cell population. However, during the IRIS event, fundamental shifts occur in HIV populations; the populations responsible for pre-therapy viremia are no longer well represented in the long-term persistent HIV populations. It is also possible that immune activation during IRIS contributes to elimination of some of these cells.

Our studies investigated genetic composition of HIV populations and have not evaluated the replication competence of the variants present. Nevertheless, the substantial shift in composition suggests effects on replication competent HIV may occur as well. Previously, Abrahams and colleagues (52) demonstrated with quantitative viral outgrowth assays (QVOA), that for most individuals initiating cART with chronic HIV infection, the long-term replication competent HIV reservoir largely reflected the virus producing population that was actively replicating at the time of cART initiation. However, for several of their study participants, the long-term viral reservoir was not the predominant replication competent reservoir. It is possible that clinical events such as the ones experienced by our study participants, shifted the composition of the virus producing HIV reservoir that persists with prolonged cART. Similar to the study participants examined by Abrahams, et al, our TBI study participant, P13, had chronic HIV infection before starting CART. When we characterized the viral populations that rebounded with CART cessation, we determined that the predominant lineage in rebound viremia did not cluster with the majority of HIV infected cell lineages detected during IRIS or after long-term cART but was also shifted from pre-therapy viral populations. This finding supports the theory that IRIS could be a clinical event experienced by chronically infected individuals starting cART, that can impact the composition of the HIV reservoir. Alternatively, productively infected cells that are activated in QVOA are more limited than our approach, so that activated provirus does not adequately represent those lineages responsible for rebound viremia in vivo.

Overall, TB associated IRIS was shown to have a profound and persistent impact on shaping HIV proviral populations during CART. Long-term persistence of HIV infected cells during CART is the principal obstacle limiting HIV eradication in infected individuals. The forces responsible for establishing the HIV reservoir after CART initiation are not well understood. Clonal expansion of HIV infected cells begins early after initial HIV infection (53) and HIV infected cell clones persist for prolonged periods (19, 39, 54-57). The data presented here indicate that the HIV reservoir in advanced HIV/AIDS has a complex structure that can be shaped by clinical events that are driven by co-infections and inflammatory responses, which can 
alter cell trafficking and power oligoclonal expansions. These observations highlight the fundamental role of co-infections in persistent HIV reservoirs that could be further explored in the design of scalable remission strategies.

\section{Materials And Methods}

\section{Study participants and sampling}

This study is comprised of sub-groups of people with sample availability enrolled in two prospective $\mathrm{NIH}$ institutional review board approved protocols: Immune reconstitution syndrome in HIV-infected patients initiating antiretroviral therapy (IRIS) (NCT00286767) (21) and PET imaging and lymph node biopsy in patients with AIDS starting antiretroviral therapy protocol (PANDORA) (NCT02147405) (22). Informed consent was obtained from all study participants before any study procedures and human subjects protection oversight was provided by the NIAID Institutional Review Board (FWA00005897). Entry criteria included: CD4 counts $<100 \mathrm{~T}$ cells $/ \mu \mathrm{L}$ and no prior antiretroviral therapy.

Pre-therapy plasma and PBMC were collected from study participants with active TB disease prior to therapy initiation, who maintained plasma HIV RNA levels at $<40$ copies $/ \mathrm{mL}$ for at least 40 weeks of CART ( $N=18$ participants). Nine of these participants developed paradoxical TB associated IRIS (TBI) after starting cART and 9 did not develop IRIS (TBNI). We also collected plasma and cells from a convenience sample of 10 study participants without a diagnosis of active TB disease and no development of IRIS (NTBNI).

\section{Soluble blood biomarkers}

Cryopreserved plasma from 25 of the study participants at baseline and 2-4 weeks after antiretroviral therapy was used for the measurement of soluble biomarkers. Plasma levels of C-reactive protein (CRP), interferon gamma (IFN-g), tumor necrosis factor (TNF-a), myeloperoxidase (MPO), interleukin (IL) 1b, IL-2, IL-6, IL-8, IL-10, IL-12p70, IL-17, IP-10, GM-CSF were measured using a custom multiplex kit by electrochemiluminescence (Meso Scale Discovery, Gaithersburg, MD). D-dimer was measured by enzyme-linked fluorescent assay on a VIDAS instrument (bioMerieux, Durham, NC). Tissue factor (TF), soluble (s) CD14 (R\&D Systems, Minneapolis, MN), sCD163 (Aviscera Bioscience, Santa Clara, CA), hyaluronic acid (HA) (Corgenix, Broomfield, CO), and hepcidin (MyBioSource, San Diego, CA) were measured using enzymelinked immunosorbent assay kits.

\section{Genomic extractions}

Viral RNA was extracted from plasma and cell culture media (58). Cell-associated (CA) RNA was extracted from purified protein derivative (PPD) stimulated cells FACS sorted into naïve (CD45RO-) and memory (CD45RO+) CD4+ cell aliquots. CA-DNA was extracted from PBMC, CD4+/CD45RO+/IFN+ cells, CD4+/CD45RO+/IFN- cells, and CD4+/CD45RO-/IFN- cells. Nucleic acid extractions were performed as described by Simonetti, et al (39). 


\section{Complimentary DNA (CDNA) synthesis}

cDNA was generated with a previously described gene specific primer (59) and Superscript IV. cDNA was synthesized from RNA inputs of $5,000,10,000$ or 20,000 , which was calculated from the HIV RNA viral load of that sampling date.

\section{Cell-associated (CA) HIV measurements}

$50 \%$ of extracted CA DNA and CA RNA was used for CA-HIV gag quantifications (37) and reported as HIV1 copies per million cells based on the CCR5 DNA assay of Thomas et al (60) using two CCR5 copies per diploid genome. The other $50 \%$ of genomic DNA was used for single genome sequencing (SGS) described in the next section. CD4 percent measured in the study protocols at each sampling time point per patient were used to normalize CA HIV levels per million CD4+ T cells and CA HIV levels per milliliter $(\mathrm{mL})$ of blood were calculated from the cell counts of each sample. CA HIV RNA:DNA ratios (steady-state HIV RNA production from cell populations) were derived from CA HIV RNA and DNA measurements.

\section{$T B$ antigen stimulations and gating strategies for CD4+ $T$ cell sorting}

Cryopreserved PBMC were obtained from two study participants from the TB-IRIS subgroup: Patient 6 at week 12 and week 96 post ART timepoints and Patient 7 at week 144 post cART timepoint. 200x106 PBMCs from the respective timepoints were thawed and stimulated with tuberculin purified protein derivative (PPD, Statens Serum Institut, Denmark) in the presence of anti- CD28/49d (BD) for 4 hours at $37^{\circ} \mathrm{C}$ and $5 \% \mathrm{CO}_{2}$ as described by $\mathrm{Hsu}$, et al (61). Cells were pelleted and the supernatant was stored at $-80^{\circ} \mathrm{C}$. CD $4+\mathrm{T}$ cells were isolated by immunomagnetic negative selection beads using an EasySep Human CD4+ T cell Isolation Kit (STEMCELL). Following CD4+ T cell isolation, IFNg secreting cells were labeled using an IFN-g Secretion Assay Detection kit in PE (Miltenyi Biotec). After IFN-g labeling, the cells were immunophenotyped with the following fluorochrome conjugated antibodies: Invitrogen Live/Dead Fixable Blue Dead Cell Stain Kit from Thermo Fisher, anti-CD3 V500 (Clone UCHT1) from BD Horizon, antiCD8 APC (Clone: SK1) from BD Biosciences, anti-CD4BV605 (Clone: RPA-T4) from BD Horizon, antiCD45RO PeCy7 (Clone: UCHL-1) from BD Biosciences. Cells were then sorted with a BD FACS Aria cell sorter on IFNg+ and IFNg- producing CD4+ T cells, and IFNg + and CD4+ T cells were further sorted on CD45RO. Sorted cells were cryopreserved in liquid nitrogen.

\section{Single Genome Sequencing (SGS)}

cDNA synthesized from viral plasma RNA and $50 \%$ of extracted CA-DNA described in the previous sections were used for SGS of $p 6 p o l(59)$ for 28 study participants.

\section{Population genetic analyses}

Sequences which were terminally mutated by APOBEC3G/F cytidine deaminase activity (hypermutated sequences; HMs) were determined with Hypermut 2.0 using consensus CDNA sequences of SGS populations derived from patient specific pre-ART viral plasma populations as the reference sequence. 
HMs were excluded from all sequence analyses. HIV subtypes were determined for all single genome sequences with the REGA HIV-1 Automated Subtyping Tool (62). Pairwise distances of HIV DNA and CDNA populations were determined and neighbor joining phylogenetic trees were constructed with MEGA 7 (63). Identical sequences were detected with ElimDupes tool provided by the Los Alamos National Laboratory http://www.hiv.lanl.gov/ (64). The likelihood that identical CA HIV DNA single genome sequences were derived from clonally expanded HIV infected cells was determined with clonal prediction scores (49) for chronic infection and with the PCR primers used for SGS (65).

We used non-parametric (46) and parametric tests (66) to determine the extent of panmixia and structural compartmentalization between HIV populations respectively. Non-parametric analyses were performed with and without identical sequences in each population to determine if identical sequences and/or changes in genetic composition of HIV populations accounted for differences between populations. For P7, we obtained three times more plasma HIV RNA derived single genomes ( $N=90$ sequences) compared with PBMC HIV DNA sequences (average of 30 sequences per sample per time point) (Supplementary Figure 9), therefore we randomly selected a comparable number (30 single genomes) 10 times without replacement, and repeated population shift tests. We obtained 4, 7 and 9 HIV DNA sequences after longterm cART from P4, P8 and P26 PBMC, which was below the threshold of 11 sequences needed to reliably predict population shifts and compartmentalization after long-term cART (46).

\section{$T$ cell receptor repertoire}

Cryopreserved PBMCs were obtained from study participants (7 NTBNI, 6 TBNI, 8 TBI) at long-term postART timepoints (weeks 64 to week 192 post cART). PBMCs were depleted of CD8+ T cells by immunomagnetic positive selection using an EasySep Human CD8+ positive selection kit (STEMCELL Technologies). CD8-depleted cell pellets were subjected to genomic DNA extraction and PCR amplification followed by immune-sequencing of the CDR3 of human TCR $\beta$ chains using the immunoSEQâ Assay (Adaptive Biotechnologies). Clonality was determined based on sequences obtained and represented with values ranging from $0-1$. The clonality value is the inverse of the normalized version of Shannon's entropy and is a measurement of the shape of the frequency distribution of all productive clones in the sample. A clonality value of 0 represents an even distribution of frequency, indicating a diverse pool of TCR clones. A clonality value of 1 represents asymmetric distribution with a few clones represented at high frequency.

\section{HIV flow assay}

HIV Flow (44) was adapted with inclusion of PPD antigen stimulation as follows: $1-2 \times 10^{8}$ cryopreserved PBMC per participant were thawed in supplemented RPMI medium containing $10 \% \mathrm{HAB}$ and $0.5 \mathrm{ul} / \mathrm{ml}$ benzonase. $C D 4+T$ cells and antigen-presenting cells were enriched from PBMCs resuspended in PBS/2\% FBS/1 mM EDTA using antibody capture of CD19 (StemCell Easy Sep Cat \#17854), CD8 (StemCell \#17853), and CD56 (StemCell \#17855). Cells were then stimulated in 6-well plates at $2 \times 10^{6}$ cells/ml with Dynabeads Human T-Activator (ThermoFisher \#111.31D, according to manufacturer 
instructions), $20 \mathrm{ng} / \mathrm{ml}$ of PPD with $1 \mathrm{ul} / 100 \mathrm{ml}$ of Fastlmmune anti-CD28/anti-CD 49d for co-stimulation (BD 347690), or no stimulation (anti-CD28/anti-CD49d alone). IL-2 was maintained throughout at a final concentration of $300 \mathrm{U} / \mathrm{ml}$ for all conditions. After 36 hours of stimulation, cells were stained with L/D aqua (ThermoFisher \#L34957) diluted 1:1000 in PBS and fixed with eBiosciences Foxp3 fix/perm buffer. Cells were stained intracellularly with $1 \mu \mathrm{L}$ per $10^{8}$ cells each of CD3-BUV395 (BD 564001), CD4-PE-Cy7 (eBio 25-2249-42), CD8-FITC (BioLegend 334704), p24 KC57-PE (Beckman Coulter 6604667), and p24 28B7-APC (MediMabs MM-0289-APC). The latter two antibodies were first reconstituted according to manufacturers' instructions and diluted 1:10 immediately prior to use. CD4+ cells were washed and acquired on a BD Fortessa flow cytometer.

\section{Statistics}

Kruskal-Wallis tests with Dunn corrections using TBI as the reference group and Ordinary one-way ANOVA tests with Bonferroni corrections were used to assess statistically significant differences among study groups and sampling time points respectively. Fisher Exact tests were used to determine if there were non-random associations between variables. Spearman-rank correlation coefficients were used to determine the relationship between measured variables in each study group. Linear mixed-effects models were used to compare longitudinal trends between study groups. Wilcoxon-Rank tests were used to determine differences in inducible virus within experiments.

\section{Declarations}

Acknowledgements: We thank Dr. Nicolas Chomont at the Department of Microbiology, Infectiology and Immunology at the Université de Montréalfor his technical suggestions for HIV Flow. We thank Nuha Naqvi and Michael Bale for their assistance in the laboratory.

Funding: This project was supported in part by the intramural research program of NIAID/NIH, with federal funds from the National Cancer Institute, National Institutes of Health, under contract numbers HHSN261200800001E and 75N91019D00024.

Author contributions: IS, FM and CML conceived and planned the experiments. IS headed the study protocols and managed study participants and sampling with MM, VS and GR. CML, MM, NA, RG, KH, CG, CC, LP, EL, AR, JB, JA and WS contributed to sample preparations and carried out the experiments. CML and $A M O$ and $M N$ performed statistical analyses and simulations. IS, FM CML, MM and RG contributed to the interpretation of the results. CML took the lead in writing the manuscript with support from IS, FM and RG. All authors provided critical feedback and helped shape the research, analysis and manuscript.

Competing Interests: The content of this publication does not necessarily reflect the views or policies of the Department of Health and Human Services, nor does mention of trade names, commercial products, or organizations imply endorsement by the U.S. Government. 
Data and materials availability: Single genome sequences have been uploaded to Genebank with accession numbers XXXXX through YYYYY.

\section{References}

1. W. World Health Organization, WHO HIV update: global epidemic, progress in scale up and policy uptake. (2019).

2. M. A. Kendall, M. Nyirenda, X. Wu, P. Ive, C. A. Benson, J. W. Andersen, S. Swindells, I. M. Sanne, D. V. Havlir, J. Kumwenda, A. C. T. G. A. S. T. Adult, Tuberculosis Immune Reconstitution Inflammatory Syndrome in A5221 STRIDE: Timing, Severity and Implications for HIV-TB programs. Journal of acquired immune deficiency syndromes (1999) 65, 423-428 (2014).

3. G. Breton, X. Duval, C. Estellat, X. Poaletti, D. Bonnet, D. Mvondo Mvondo, P. Longuet, C. Leport, J. L. Vilde, Determinants of immune reconstitution inflammatory syndrome in HIV type 1-infected patients with tuberculosis after initiation of antiretroviral therapy. Clin Infect Dis 39, 1709-1712 (2004).

4. R. A. M. Breen, C. J. Smith, H. Bettinson, S. Dart, B. Bannister, M. A. Johnson, M. C. I. Lipman, Paradoxical reactions during tuberculosis treatment in patients with and without HIV co-infection. Thorax 59, 704 (2004).

5. C. Michailidis, S. Pozniak Al Fau - Mandalia, S. Mandalia S Fau - Basnayake, M. R. Basnayake S Fau Nelson, B. G. Nelson Mr Fau - Gazzard, B. G. Gazzard, Clinical characteristics of IRIS syndrome in patients with HIV and tuberculosis. Antivir Ther 10, 417-422.

6. S. D. Lawn, L.-G. Myer L Fau - Bekker, R. Bekker Lg Fau - Wood, R. Wood, Tuberculosis-associated immune reconstitution disease: incidence, risk factors and impact in an antiretroviral treatment service in South Africa. AIDS 21, 335-341 (2007).

7. W. World Health Organization, Global Tuberculosis Report 2017. 63 (2017).

8. G. Meintjes, C. Stek, L. Blumenthal, F. Thienemann, C. Schutz, J. Buyze, R. Ravinetto, H. van Loen, A. Nair, A. Jackson, R. Colebunders, G. Maartens, R. J. Wilkinson, L. Lynen, Prednisone for the Prevention of Paradoxical Tuberculosis-Associated IRIS. New England Journal of Medicine 379, 1915-1925 (2018).

9. P. E. Namale, L. H. Abdullahi, S. Fine, M. Kamkuemah, R. J. Wilkinson, G. Meintjes, Paradoxical TBIRIS in HIV-infected adults: a systematic review and meta-analysis. Future Microbiology 10, $1077-$ 1099 (2015).

10. E. Bruzzesi, I. Sereti, Residual Immune Activation and Latency. Curr Top Microbiol Immunol 417, 157180 (2018).

11. I. Sereti, Immune reconstruction inflammatory syndrome in HIV infection: beyond what meets the eye. Top Antivir Med 27, 106-111 (2020).

12. L. R. Antonelli, J. N. Mahnke Y Fau - Hodge, B. O. Hodge Jn Fau - Porter, D. L. Porter Bo Fau - Barber, R. Barber DI Fau - DerSimonian, J. H. DerSimonian R Fau - Greenwald, G. Greenwald Jh Fau - Roby, J. Roby G Fau - Mican, A. Mican J Fau - Sher, M. Sher A Fau - Roederer, I. Roederer M Fau - Sereti, I. 
Sereti, Elevated frequencies of highly activated CD4+ T cells in HIV+ patients developing immune reconstitution inflammatory syndrome. Blood 116, (2010).

13. O. Goovaerts, W. Jennes, M. Massinga-Loembé, A. Ceulemans, W. Worodria, H. Mayanja-Kizza, R. Colebunders, L. Kestens, T. B. I. S. G. the, LPS-Binding Protein and IL-6 Mark Paradoxical Tuberculosis Immune Reconstitution Inflammatory Syndrome in HIV Patients. PLoS ONE 8, e81856 (2013).

14. S. Ravimohan, N. Tamuhla, K. Nfanyana, A. P. Steenhoff, R. Letlhogile, I. Frank, R. R. MacGregor, R. Gross, D. Weissman, G. P. Bisson, Robust Reconstitution of Tuberculosis-Specific Polyfunctional CD4 (+) T-Cell Responses and Rising Systemic Interleukin 6 in Paradoxical Tuberculosis-Associated Immune Reconstitution Inflammatory Syndrome. Clinical Infectious Diseases: An Official Publication of the Infectious Diseases Society of America 62, 795-803 (2016).

15. A. Bourgarit, V. Carcelain G Fau - Martinez, C. Martinez V Fau - Lascoux, V. Lascoux C Fau - Delcey, B. Delcey V Fau - Gicquel, E. Gicquel B Fau - Vicaut, P. H. Vicaut E Fau - Lagrange, D. Lagrange Ph Fau Sereni, B. Sereni D Fau - Autran, B. Autran, Explosion of tuberculin-specific Th1-responses induces immune restoration syndrome in tuberculosis and HIV co-infected patients. AIDS 20, (2006).

16. A. Bourgarit, A. Carcelain G Fau - Samri, C. Samri A Fau - Parizot, M. Parizot C Fau - Lafaurie, S. Lafaurie M Fau - Abgrall, V. Abgrall S Fau - Delcey, E. Delcey V Fau - Vicaut, D. Vicaut E Fau - Sereni, B. Sereni D Fau - Autran, B. Autran, Tuberculosis-associated immune restoration syndrome in HIV-1infected patients involves tuberculin-specific CD4 Th1 cells and KIR-negative gammadelta T cells. Journal of Immunology 183, 3915-3923 (2009).

17. D. B. Tan, H. Y. Yong Yk Fau - Tan, A. Tan Hy Fau - Kamarulzaman, L. H. Kamarulzaman A Fau - Tan, A. Tan Lh Fau - Lim, I. Lim A Fau - James, M. James I Fau - French, P. French M Fau - Price, P. Price, Immunological profiles of immune restoration disease presenting as mycobacterial lymphadenitis and cryptococcal meningitis. HIV Med 9, 307-316 (2008).

18. R. Vignesh, N. Kumarasamy, A. Lim, S. Solomon, K. G. Murugavel, P. Balakrishnan, S. S. Solomon, K. H. Mayer, C. R. Swathirajan, E. Chandrasekaran, A. Pradeep, S. Poongulali, C. A. Benson, M. A. French, TB-IRIS after initiation of antiretroviral therapy is associated with expansion of preexistent Th1 responses against Mycobacterium tuberculosis antigens. J Acquir Immune Defic Syndr 64, 241-248 (2013).

19. G. Bozzi, F. R. Simonetti, S. A. Watters, E. M. Anderson, M. Gouzoulis, M. F. Kearney, P. Rote, C. Lange, W. Shao, R. Gorelick, B. Fullmer, S. Kumar, S. Wank, S. Hewitt, D. E. Kleiner, J. Hattori, M. J. Bale, S. Hill, J. Bell, C. Rehm, Z. Grossman, R. Yarchoan, T. Uldrick, F. Maldarelli, No evidence of ongoing HIV replication or compartmentalization in tissues during combination antiretroviral therapy: Implications for HIV eradication. Sci Adv 5, eaav2045 (2019).

20. A. C. T. G. Network, AIDS Clinical Trials Group Network (ACTG) definition of immune reconstitution inflammatory syndrome (IRIS). (2009).

21. C. g. I. B. (MD), Identifier NCT00286767 - Immune Reconstitution Syndrome in HIV-Infected Patients Taking Antiretroviral Therapy (IRIS). National Library of Medicine (US), (October 7, 2019). 
22. C. g. I. B. (MD), Identifier NCT02147405 - PET Imaging and Lymph Node Assessment of IRIS in People With AIDS (PANDORA). National Library of Medicine (US), (December 9, 2019).

23. V. Bouteloup, C. Sabin, A. Mocroft, L. Gras, N. Pantazis, V. Le Moing, A. d'Arminio Monforte, M. MaryKrause, B. Roca, J. M. Miro, M. Battegay, N. Brockmeyer, J. Berenguer, P. Morlat, N. Obel, S. De Wit, G. Fatkenheuer, R. Zangerle, J. Ghosn, S. Perez-Hoyos, M. Campbell, M. Prins, G. Chene, L. Meyer, M. Dorrucci, C. Torti, R. Thiebaut, C. D. R. t. H. P. T. f. t. C. o. O. H. I. V. E. R. E. i. E. Standard Reference Distribution of, Reference curves for CD4 T-cell count response to combination antiretroviral therapy in HIV-1-infected treatment-naive patients. HIV Med 18, 33-44 (2017).

24. H. M. Soeters, S. Napravnik, M. R. Patel, J. J. Eron, Jr., A. Van Rie, The effect of tuberculosis treatment on virologic and $C D 4+$ cell count response to combination antiretroviral therapy: a systematic review. AIDS 28, 245-255 (2014).

25. G. W. H. Organization, Guidelines for managing advanced HIV disease and rapid initiation of antiretroviral therapy. CC BY-NC-SA 3.0 IGO, (2017).

26. I. Sereti, V. Sheikh, D. Shaffer, N. Phanuphak, E. Gabriel, J. Wang, M. C. Nason, G. Roby, H. Ngeno, F. Kirui, A. Pau, J. M. Mican, A. Rupert, R. Bishop, B. Agan, N. Chomchey, N. Teeratakulpisarn, S. Tansuphaswadikul, D. Langat, J. Kosgei, M. French, J. Ananworanich, F. Sawe, Prospective international study of incidence and predictors of immune reconstitution inflammatory syndrome and death in people with HIV and severe lymphopenia. Clin Infect Dis 71, 652-660 (2019).

27. D. R. Boulware, C. E. Hullsiek Kh Fau - Puronen, A. Puronen Ce Fau - Rupert, J. V. Rupert A Fau - Baker, M. A. Baker Jv Fau - French, P. R. French Ma Fau - Bohjanen, R. M. Bohjanen Pr Fau - Novak, J. D. Novak Rm Fau - Neaton, I. Neaton Jd Fau - Sereti, I. Sereti, Higher levels of CRP, D-dimer, IL-6, and hyaluronic acid before initiation of antiretroviral therapy (ART) are associated with increased risk of AIDS or death. J Infect Dis 203, 1637-1646 (2011).

28. B. O. Porter, J. N. Ouedraogo GI Fau - Hodge, M. A. Hodge Jn Fau - Smith, A. Smith Ma Fau - Pau, G. Pau A Fau - Roby, R. Roby G Fau - Kwan, R. J. Kwan R Fau - Bishop, C. Bishop Rj Fau - Rehm, J. Rehm C Fau - Mican, I. Mican J Fau - Sereti, I. Sereti, d-Dimer and CRP levels are elevated prior to antiretroviral treatment in patients who develop IRIS. Clin Immunol 136, $42-50$ (2010).

29. T. Matsuyama, G. Hamamoto Y Fau - Soma, D. Soma G Fau - Mizuno, N. Mizuno D Fau - Yamamoto, N. Yamamoto N Fau - Kobayashi, N. Kobayashi, Cytocidal effect of tumor necrosis factor on cells chronically infected with human immunodeficiency virus (HIV): enhancement of HIV replication. $J$ Virol 63, 2504-2509 (1989).

30. E. J. Duh, T. M. Maury Wj Fau - Folks, A. S. Folks Tm Fau - Fauci, A. B. Fauci As Fau - Rabson, A. B. Rabson, Tumor necrosis factor alpha activates human immunodeficiency virus type 1 through induction of nuclear factor binding to the NF-kappa B sites in the long terminal repeat. Proc Natl Acad Sci U S A 86, 5974-5978 (1989).

31. J. Fantini, N. Bolmont C Fau - Yahi, N. Yahi, Tumor necrosis factor-alpha stimulates both apical and basal production of HIV in polarized human intestinal HT29 cells. Immunol Lett 34, 85-90. 
32. X. X. Ren, L. Ma, W. W. Sun, W. D. Kuang, T. S. Li, X. Jin, J. H. Wang, Dendritic cells maturated by coculturing with HIV-1 latently infected Jurkat T cells or stimulating with AIDS-associated pathogens secrete TNF-alpha to reactivate HIV-1 from latency. Virulence 8, 1732-1743 (2017).

33. S. Palmer, A. Maldarelli F Fau - Wiegand, B. Wiegand A Fau - Bernstein, G. J. Bernstein B Fau - Hanna, S. C. Hanna Gj Fau - Brun, D. J. Brun Sc Fau - Kempf, J. W. Kempf Dj Fau - Mellors, J. M. Mellors Jw Fau - Coffın, M. S. Coffin Jm Fau - King, M. S. King, Low-level viremia persists for at least 7 years in patients on suppressive antiretroviral therapy. Proc Natl Acad Sci USA 105, 3879-3884.

34. A. S. Perelson, P. Essunger, Y. Cao, M. Vesanen, A. Hurley, K. Saksela, M. Markowitz, D. D. Ho, Decay characteristics of HIV-1-infected compartments during combination therapy. Nature 387, 188-191 (1997).

35. M. Markowitz, M. Louie, A. Hurley, E. Sun, M. Di Mascio, A. S. Perelson, D. D. Ho, A novel antiviral intervention results in more accurate assessment of human immunodeficiency virus type 1 replication dynamics and T-cell decay in vivo. J Virol 77, 5037-5038 (2003).

36. A. Andrade, S. L. Rosenkranz, A. R. Cillo, D. Lu, E. S. Daar, J. M. Jacobson, M. Lederman, E. P. Acosta, T. Campbell, J. Feinberg, C. Flexner, J. W. Mellors, D. R. Kuritzkes, A. C. T. G. A. Team, Three distinct phases of HIV-1 RNA decay in treatment-naive patients receiving raltegravir-based antiretroviral therapy: ACTG A5248. J Infect Dis 208, 884-891 (2013).

37. M. Somsouk, R. M. Dunham, M. Cohen, R. Albright, M. Abdel-Mohsen, T. Liegler, J. Lifson, M. Piatak, R. Gorelick, Y. Huang, Y. Wu, P. Y. Hsue, J. N. Martin, S. G. Deeks, J. M. McCune, P. W. Hunt, The Immunologic Effects of Mesalamine in Treated HIV-Infected Individuals with Incomplete CD4+ T Cell Recovery: A Randomized Crossover Trial. PLOS ONE 9, e116306 (2015).

38. G. J. Besson, C. M. Lalama, R. J. Bosch, R. T. Gandhi, M. A. Bedison, E. Aga, S. A. Riddler, D. K. McMahon, F. Hong, J. W. Mellors, HIV-1 DNA decay dynamics in blood during more than a decade of suppressive antiretroviral therapy. Clin Infect Dis 59, 1312-1321.

39. F. R. Simonetti, M. D. Sobolewski, E. Fyne, W. Shao, J. Spindler, J. Hattori, E. M. Anderson, S. A. Watters, S. Hill, X. Wu, D. Wells, L. Su, B. T. Luke, E. K. Halvas, G. Besson, K. J. Penrose, Z. Yang, R. W. Kwan, C. Van Waes, T. Uldrick, D. E. Citrin, J. Kovacs, M. A. Polis, C. A. Rehm, R. Gorelick, M. Piatak, B. F. Keele, M. F. Kearney, J. M. Coffin, S. H. Hughes, J. W. Mellors, F. Maldarelli, Clonally expanded CD4+ T cells can produce infectious HIV-1 in vivo. Proc Natl Acad Sci USA 113, 1883-1888.

40. Y. D. Mahnke, R. Greenwald Jh Fau - DerSimonian, G. DerSimonian R Fau - Roby, L. R. V. Roby G Fau Antonelli, A. Antonelli Lr Fau - Sher, M. Sher A Fau - Roederer, I. Roederer M Fau - Sereti, I. Sereti, Selective expansion of polyfunctional pathogen-specific CD4 (+) T cells in HIV-1-infected patients with immune reconstitution inflammatory syndrome. Blood 119, 3105-3112.

41. E. M. Anderson, F. R. Simonetti, R. J. Gorelick, S. Hill, M. A. Gouzoulis, J. Bell, C. Rehm, L. Perez, E. Boritz, X. Wu, D. Wells, S. H. Hughes, V. Rao, J. M. Coffin, M. F. Kearney, F. Maldarelli, Dynamic Shifts in the HIV Proviral Landscape During Long Term Combination Antiretroviral Therapy: Implications for Persistence and Control of HIV Infections. Viruses 12, (2020). 
42. M. J. Peluso, P. Bacchetti, K. D. Ritter, S. Beg, J. Lai, J. N. Martin, P. W. Hunt, T. J. Henrich, J. D. Siliciano, R. F. Siliciano, G. M. Laird, S. G. Deeks, Differential decay of intact and defective proviral DNA in HIV-1-infected individuals on suppressive antiretroviral therapy. JCI Insight 5, (2020).

43. G. Meintjes, K. A. Wilkinson, M. X. Rangaka, K. Skolimowska, K. van Veen, M. Abrahams, R. Seldon, D. J. Pepper, K. Rebe, P. Mouton, G. van Cutsem, M. P. Nicol, G. Maartens, R. J. Wilkinson, Type 1 helper $T$ cells and FoxP3-positive $T$ cells in HIV-tuberculosis-associated immune reconstitution inflammatory syndrome. Am J Respir Crit Care Med 178, 1083-1089 (2008).

44. M. Pardons, A. E. Baxter, M. Massanella, A. Pagliuzza, R. Fromentin, C. Dufour, L. Leyre, J. P. Routy, D. E. Kaufmann, N. Chomont, Single-cell characterization and quantification of translation-competent viral reservoirs in treated and untreated HIV infection. PLoS Pathog 15, e1007619 (2019).

45. F. Maldarelli, S. Kearney M Fau - Palmer, R. Palmer S Fau - Stephens, J. Stephens R Fau - Mican, M. A. Mican J Fau - Polis, R. T. Polis Ma Fau - Davey, J. Davey Rt Fau - Kovacs, W. Kovacs J Fau - Shao, D. Shao W Fau - Rock-Kress, J. A. Rock-Kress D Fau - Metcalf, C. Metcalf Ja Fau - Rehm, S. E. Rehm C Fau - Greer, D. L. Greer Se Fau - Lucey, K. Lucey DI Fau - Danley, H. Danley K Fau - Alter, J. W. Alter H Fau - Mellors, J. M. Mellors Jw Fau - Coffin, J. M. Coffin, HIV populations are large and accumulate high genetic diversity in a nonlinear fashion. J Viro/ 87, 10313-10323.

46. G. Achaz, M. Palmer S Fau - Kearney, F. Kearney M Fau - Maldarelli, J. W. Maldarelli F Fau - Mellors, J. M. Mellors Jw Fau - Coffın, J. Coffin Jm Fau - Wakeley, J. Wakeley, A robust measure of HIV-1 population turnover within chronically infected individuals. Molecular Biology and Evolution 21, 1902-1912 (2004).

47. J. S. Mary F. Kearney , Ann Wiegand, Wei Shao, Richard Haubrich, Sharon Riddler, Christina M. Lalama, Michael D. Hughes, John M. Coffin, John W. Mellors, Lower pre-ART intra-participant HIV-1 pol diversity may not be associated with virologic failure in adults. PLOS ONE 13, (2018).

48. L. Zheng, E. S. Bosch Rj Fau - Chan, S. Chan Es Fau - Read, M. Read S Fau - Kearney, D. M. Kearney M Fau - Margolis, J. W. Margolis Dm Fau - Mellors, J. J. Mellors Jw Fau - Eron, R. T. Eron Jj Fau Gandhi, R. T. Gandhi, Predictors of residual viraemia in patients on long-term suppressive antiretroviral therapy. Antivir Ther 18, 39-43 (2013).

49. S. B. Laskey, C. W. Pohlmeyer, K. M. Bruner, R. F. Siliciano, Evaluating Clonal Expansion of HIVInfected Cells: Optimization of PCR Strategies to Predict Clonality. PLoS Pathogens 12, e1005689 (2016).

50. M. Slatkin, W. P. Maddison, A cladistic measure of gene flow inferred from the phylogenies of alleles. Genetics 123, 603-613 (1989).

51. M. A. French, N. Lenzo, M. John, S. A. Mallal, E. J. McKinnon, I. R. James, P. Price, J. P. Flexman, M. L. Tay-Kearney, Immune restoration disease after the treatment of immunodeficient HIV-infected patients with highly active antiretroviral therapy. HIV Med 1, 107-115 (2000).

52. M. R. Abrahams, S. B. Joseph, N. Garrett, L. Tyers, M. Moeser, N. Archin, O. D. Council, D. Matten, S. Zhou, D. Doolabh, C. Anthony, N. Goonetilleke, S. A. Karim, D. M. Margolis, S. K. Pond, C. Williamson, 
R. Swanstrom, The replication-competent HIV-1 latent reservoir is primarily established near the time of therapy initiation. Sci Trans/ Med 11, (2019).

53. J. M. Coffin, D. W. Wells, J. M. Zerbato, J. D. Kuruc, S. Guo, B. T. Luke, J. J. Eron, M. Bale, J. Spindler, F. R. Simonetti, S. Hill, M. F. Kearney, F. Maldarelli, X. Wu, J. W. Mellors, S. H. Hughes, Clones of infected cells arise early in HIV-infected individuals. JCI Insight 4, (2019).

54. F. Maldarelli, X. Wu, L. Su, F. R. Simonetti, W. Shao, S. Hill, J. Spindler, A. L. Ferris, J. W. Mellors, M. F. Kearney, J. M. Coffin, S. H. Hughes, HIV latency. Specific HIV integration sites are linked to clonal expansion and persistence of infected cells. Science 345, 179-183 (2014).

55. T. A. Wagner, S. McLaughlin, K. Garg, C. Y. K. Cheung, B. B. Larsen, S. Styrchak, H. C. Huang, P. T. Edlefsen, J. I. Mullins, L. M. Frenkel, Proliferation of cells with HIV integrated into cancer genes contributes to persistent infection. Science (New York, N. Y.) 345, 570-573 (2014).

56. E. D. Sverdlov, K. E. Petrukhin, N. S. Akop'iants, N. E. Broude, G. S. Monastyrskaia, [Differential expression of 2 genes of the $\mathrm{Na}+, \mathrm{K}+$-AtPase subunit in normal and tumor tissues in humans]. Dokl Akad Nauk SSSR 298, 236-239 (1988).

57. L. B. Cohn, I. T. Silva, T. Y. Oliveira, R. A. Rosales, E. H. Parrish, G. H. Learn, B. H. Hahn, J. L. Czartoski, M. J. McElrath, C. Lehmann, F. Klein, M. Caskey, B. D. Walker, J. D. Siliciano, R. F. Siliciano, M. Jankovic, M. C. Nussenzweig, HIV-1 integration landscape during latent and active infection. Cell 160, 420-432 (2015).

58. A. N. Cline, J. W. Bess, M. Piatak, Jr., J. D. Lifson, Highly sensitive SIV plasma viral load assay: practical considerations, realistic performance expectations, and application to reverse engineering of vaccines for AIDS. J Med Primato/ 34, 303-312 (2005).

59. M. F. Kearney, J. Spindler, W. Shao, S. Yu, E. M. Anderson, A. O'Shea, C. Rehm, C. Poethke, N. Kovacs, J. W. Mellors, J. M. Coffin, F. Maldarelli, Lack of detectable HIV-1 molecular evolution during suppressive antiretroviral therapy. PLoS Pathog 10, e1004010 (2014).

60. J. A. Thomas, W. G. Gagliardi Td Fau - Alvord, M. Alvord Wg Fau - Lubomirski, W. J. Lubomirski M Fau - Bosche, R. J. Bosche Wj Fau - Gorelick, R. J. Gorelick, Human immunodeficiency virus type 1 nucleocapsid zinc-finger mutations cause defects in reverse transcription and integration. Virology 353, 41-51 (2006).

61. D. C. Hsu, K. F. Faldetta, L. Pei, V. Sheikh, N. S. Utay, G. Roby, A. Rupert, A. S. Fauci, I. Sereti, A Paradoxical Treatment for a Paradoxical Condition: Infliximab Use in Three Cases of Mycobacterial IRIS. Clin Infect Dis 62, 258-261 (2016).

62. T. de Oliveira, K. Deforche, S. Cassol, M. Salminen, D. Paraskevis, C. Seebregts, J. Snoeck, E. J. van Rensburg, A. M. Wensing, D. A. van de Vijver, C. A. Boucher, R. Camacho, A. M. Vandamme, An automated genotyping system for analysis of HIV-1 and other microbial sequences. Bioinformatics 21, 3797-3800 (2005).

63. S. Kumar, G. Stecher, K. Tamura, MEGA7: Molecular Evolutionary Genetics Analysis Version 7.0 for Bigger Datasets. Mol Biol Evol 33, 1870-1874. 
64. F. B, L. T, A. C, H. B, M. I, M. J, R. A, W. S, K. B, Eds, HIV Sequence Compendium 2018. Theoretical Biology and Biophysics Group Los Alamos National Laboratory, (2018).

65. S. Palmer, F. Kearney M Fau - Maldarelli, E. K. Maldarelli F Fau - Halvas, C. J. Halvas Ek Fau - Bixby, H. Bixby Cj Fau - Bazmi, D. Bazmi H Fau - Rock, J. Rock D Fau - Falloon, R. T. Falloon J Fau - Davey, Jr., R. L. Davey Rt Jr Fau - Dewar, J. A. Dewar RI Fau - Metcalf, S. Metcalf Ja Fau - Hammer, J. W. Hammer S Fau - Mellors, J. M. Mellors Jw Fau - Coffin, J. M. Coffin, Multiple, linked human immunodeficiency virus type 1 drug resistance mutations in treatment-experienced patients are missed by standard genotype analysis. J Clin Microbio/ 43, 406-413 (2005).

66. S. L. K. Pond, S. D. W. Frost, S. V. Muse, HyPhy: hypothesis testing using phylogenies. Bioinformatics 21, 676-679 (2005).

\section{Figures}

(a)

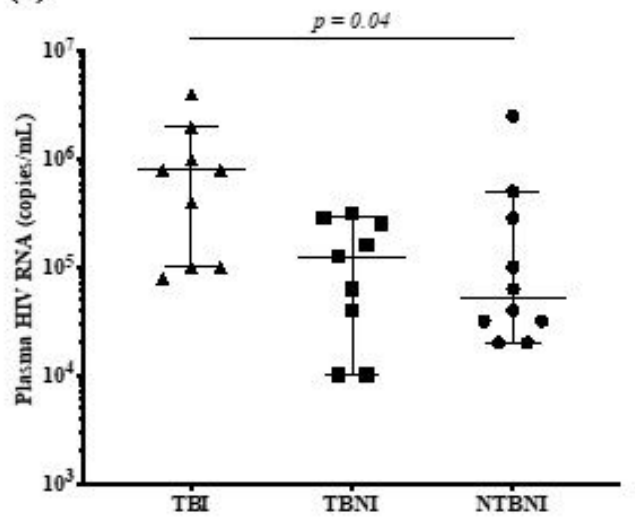

(d)

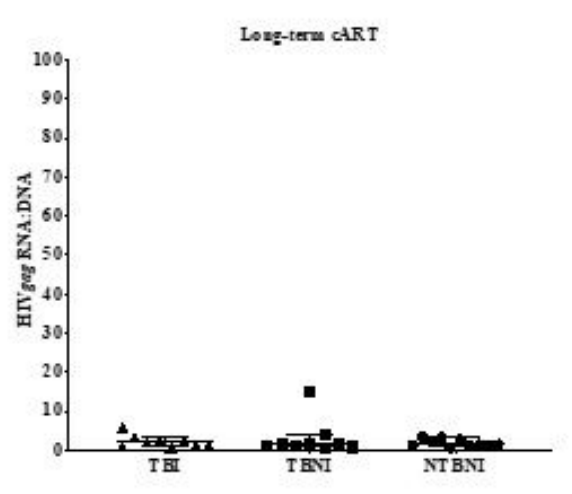

(b)

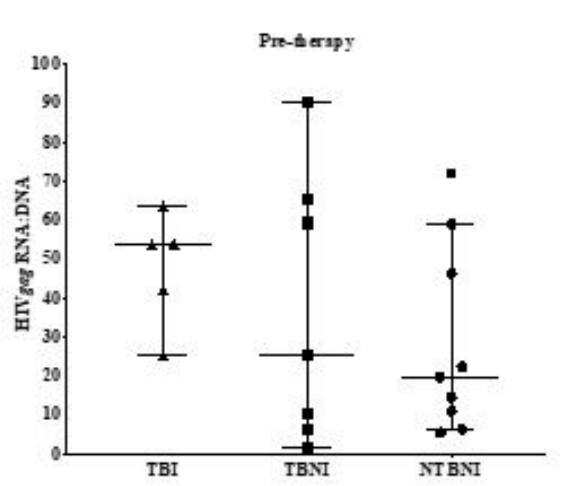

(e)

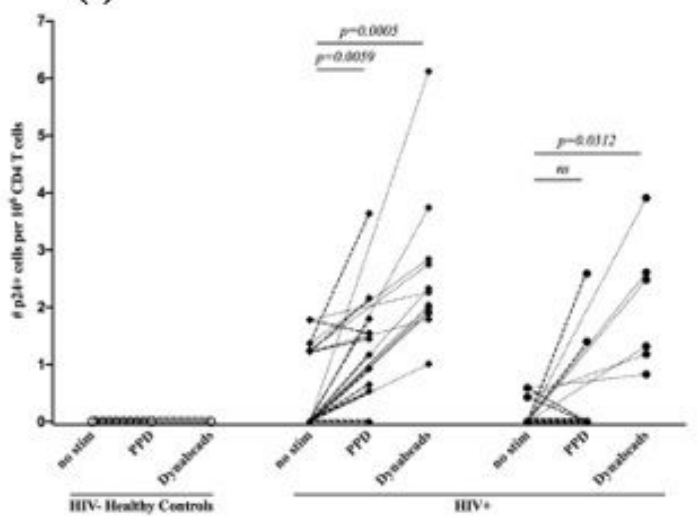

(c)

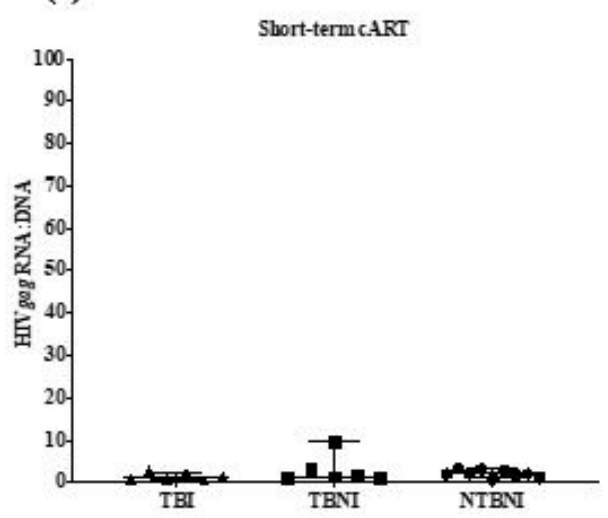

\section{Figure 1}

HIV RNA levels and TB-Ag stimulation of HIV infected cells with HIV FLOW. (a) Pre-therapy HIV RNA levels in TBI, TBNI BNI and NTBNI study participants. Cell-associated HIV RNA:DNA ratios (b) prior to cART initiation, (c) after short- term cART and (d) after long-term cART. Median and interquartile ranges are shown. $\mathrm{P}>0.05$ for all comparisons by Kruskal-Wallis tests with Dunn's corrections for multiple comparisons. (e) Proportion of 28B7+/KC57+ (p24) double-positive CD4+ T cells after PPD, dynabeads or 
no stimulation in study participants with and without HIV/ TB co- infection after long-term cART and healthy HIV negative (HIV-) controls. Significant p-values shown; Wilcoxon-rank test.



(b)

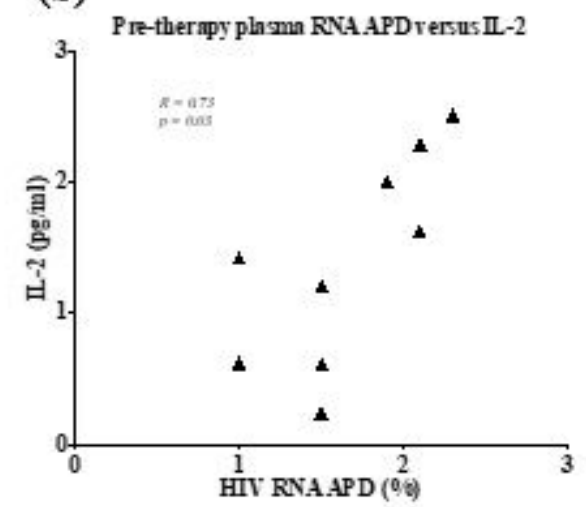

(c)

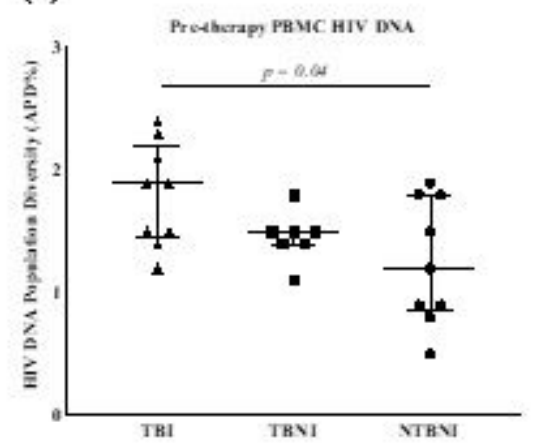

(d)

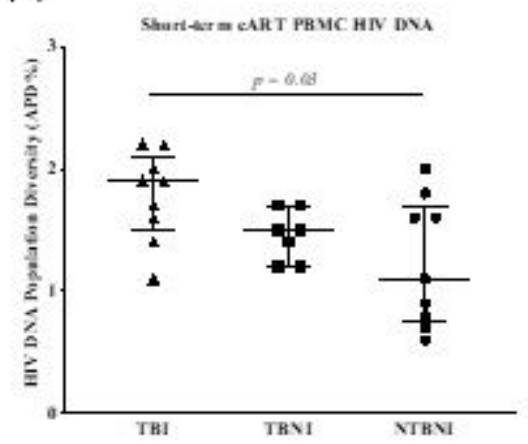

(e)

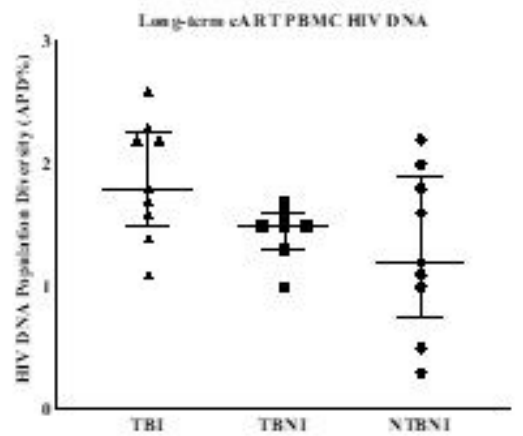

(f)

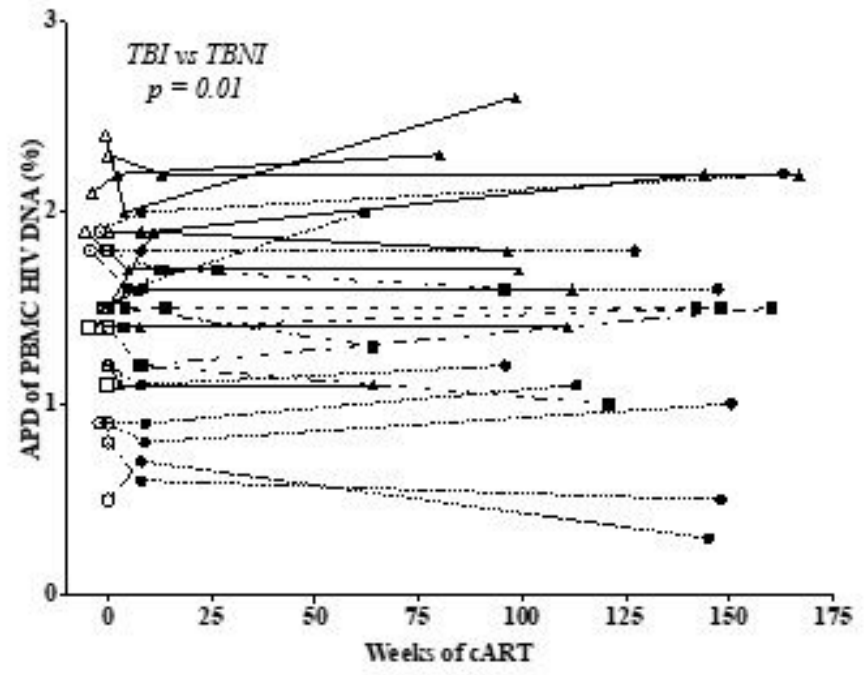

Figure 2

HIV population diversity. (a) Average pairwise distance (APD) of pre-therapy plasma HIV RNA populations among groups. (b) Spearman correlation of pre-therapy plasma HIV RNA APD (x-axis) versus IL-2 levels (y-axis) in the TBI group . APD of PBMC HIV DNA populations (c) prior to cART initiation, (d) after shortand (e) long-term cART in TBI, TBNI NTBNI. Significant p-values are shown; Kruskal-Wallis tests. (f) Temporal trends in PBMC HIV DNA APD (linear mixed-effects model). Open symbols = pretherapy populations. 
(a)

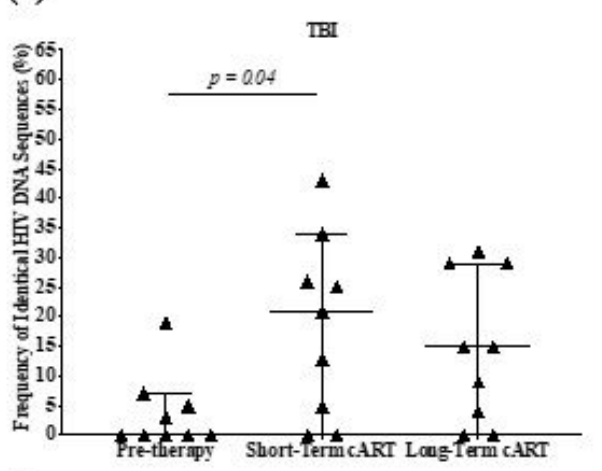

(d)

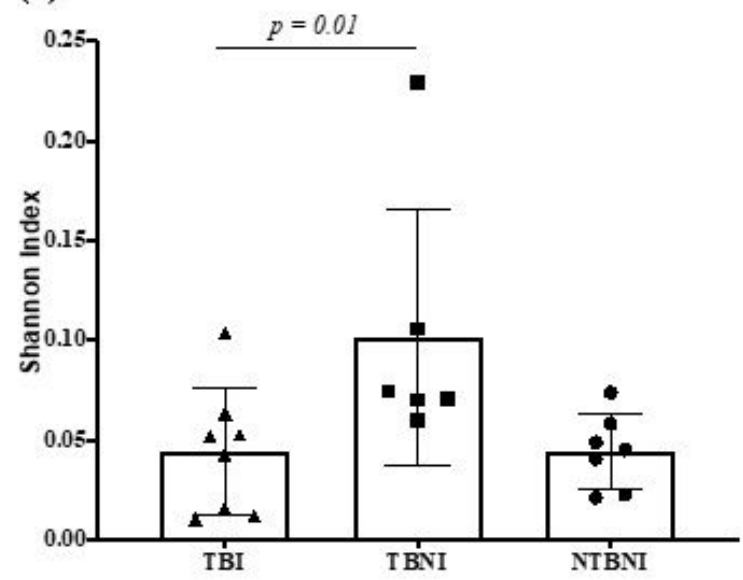

(c)

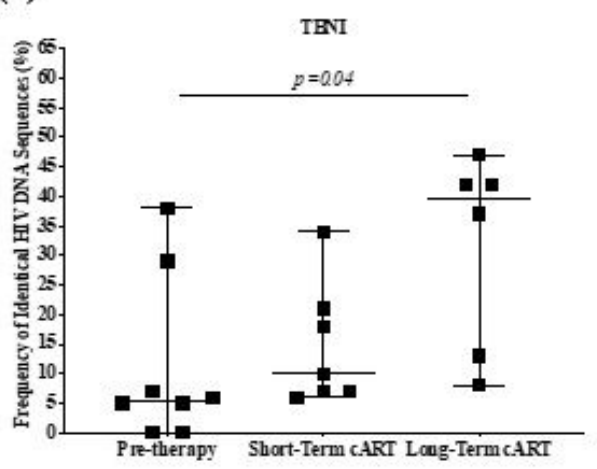

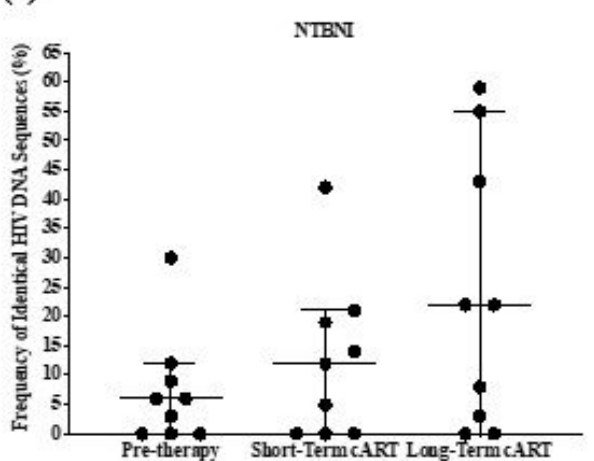

\section{Figure 3}

Phylogenetic analyses. Frequency of identical HIV DNA sequences on cART in (a) TBI, (b) TBNI and (c) NTBNI study participants. (d) Shannon Index of CD4+ T cell receptor repertoires after long-term cART. Significant p-values are shown; One-way ANOVA and Kruskal-Wallis tests respectively. 
(a)

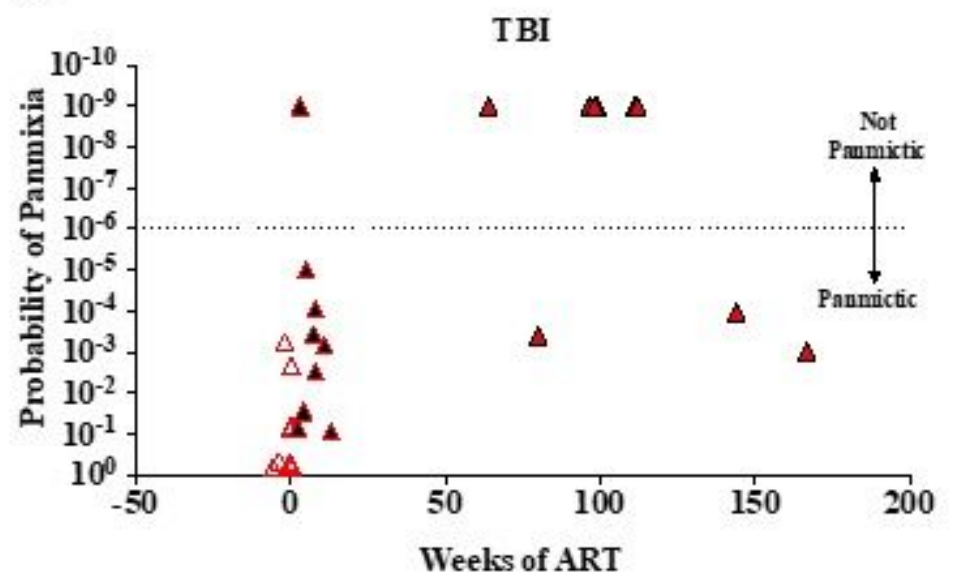

(b)

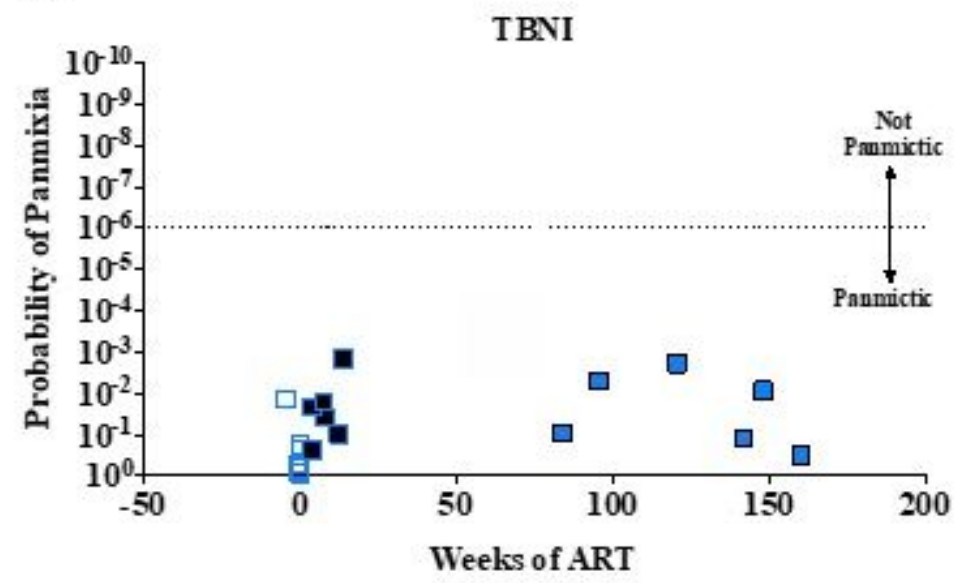

(c)

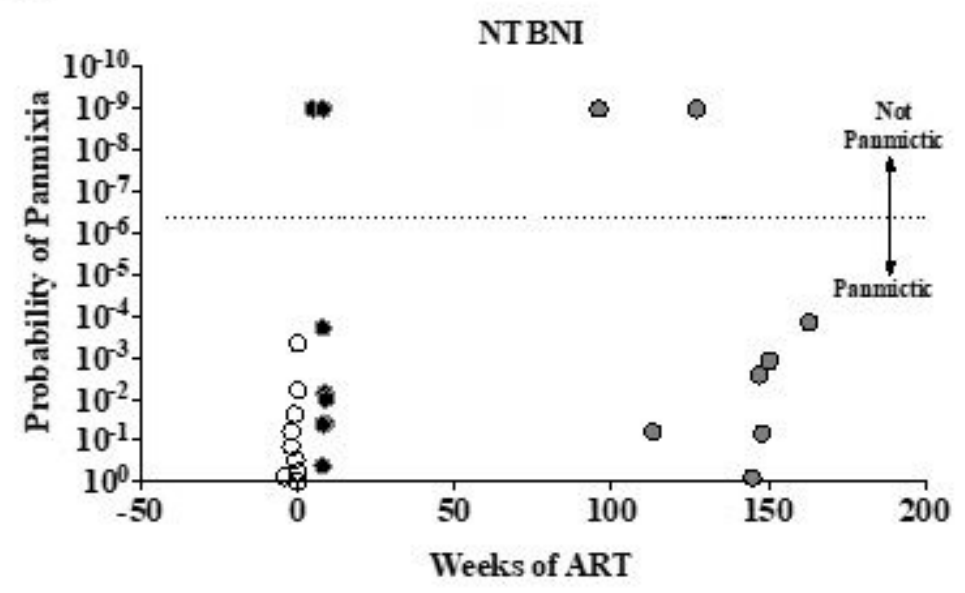

\section{Figure 4}

Probability of pre-therapy plasma HIV RNA populations being panmictic with PBMC derived HIV DNA populations prior to CART (open symbols), after short-term cART (black enclosed symbols) and after longterm cART (red, blue and grey enclosed symbols). Triangles represent TBI (a), squares represent TBNI (b), circles represent NTBNI (c). Geographic subdivision tests were performed with identical sequences in each sample represented as a single sequence to denote its lineage. 


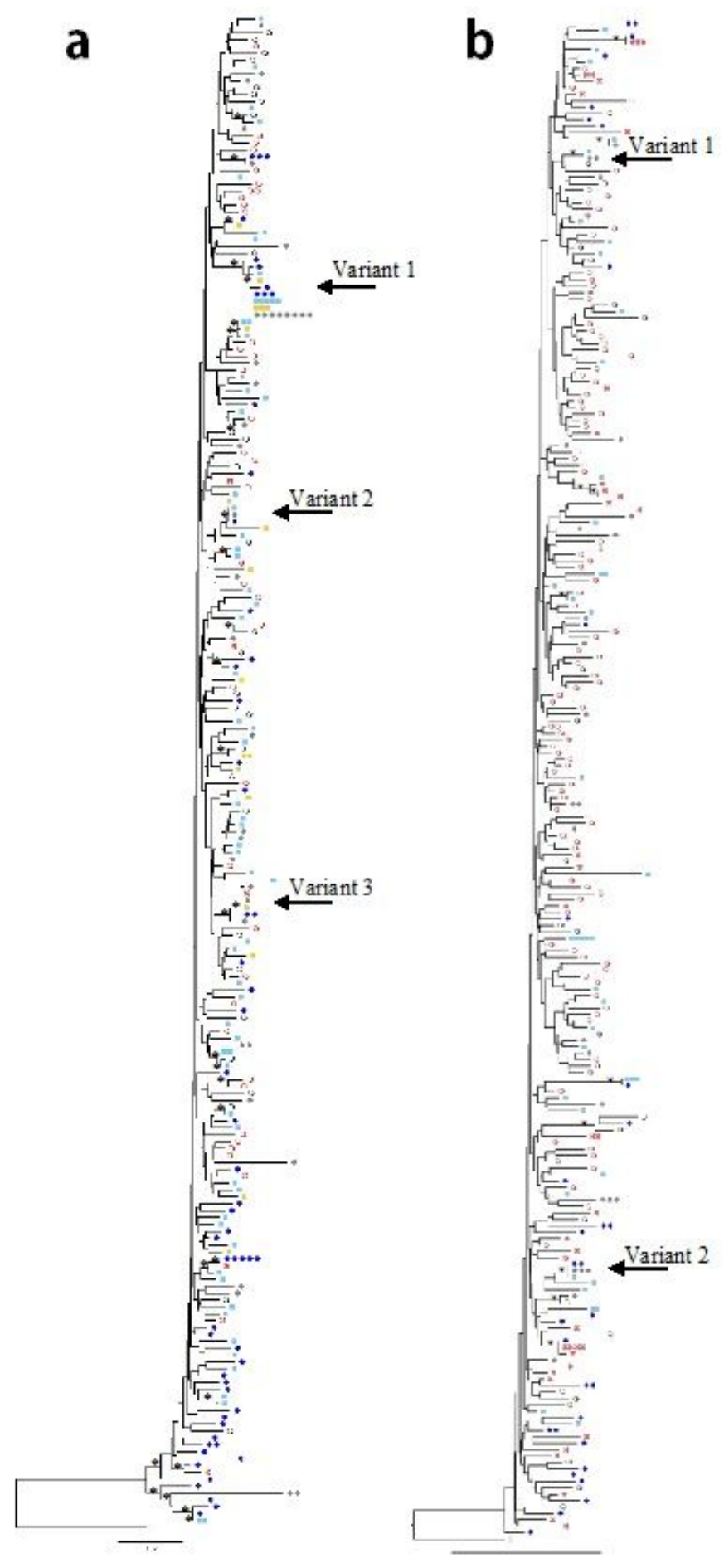

\section{Figure 5}

Neighbor-Joining tree of HIV populations in P6 (a) and P7 (b). Pre-therapy plasma HIV RNA and PBMC DNA prior to CART initiation, PBMC DNA after short- and long- term CART .CD4+ CD45RO+ IFN $\triangle+$ cells HIV DNA from P6 during IRIS and after long-term cART in P6 and P7. Supernatant HIV RNA after PPD stimulation. * bootstrap values $>70$. Arrows indicate variants responsive to PPD and detected after longterm cART. 
(a)

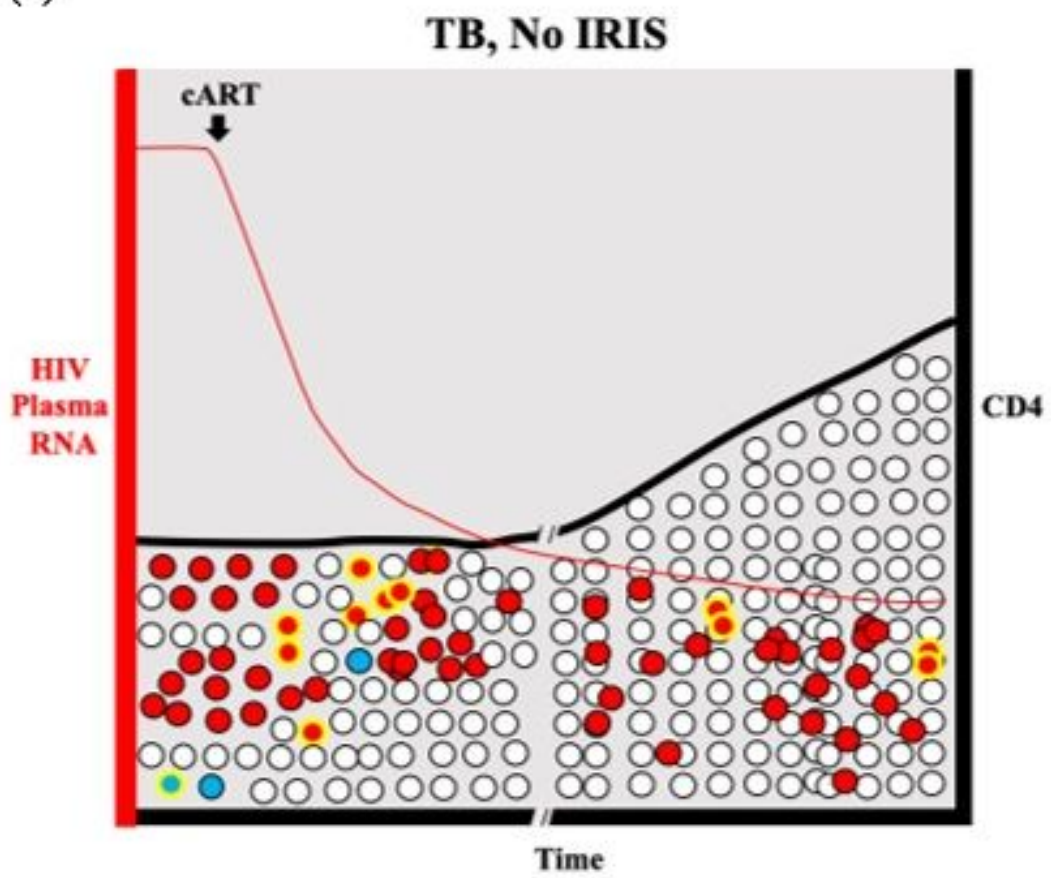

(b)

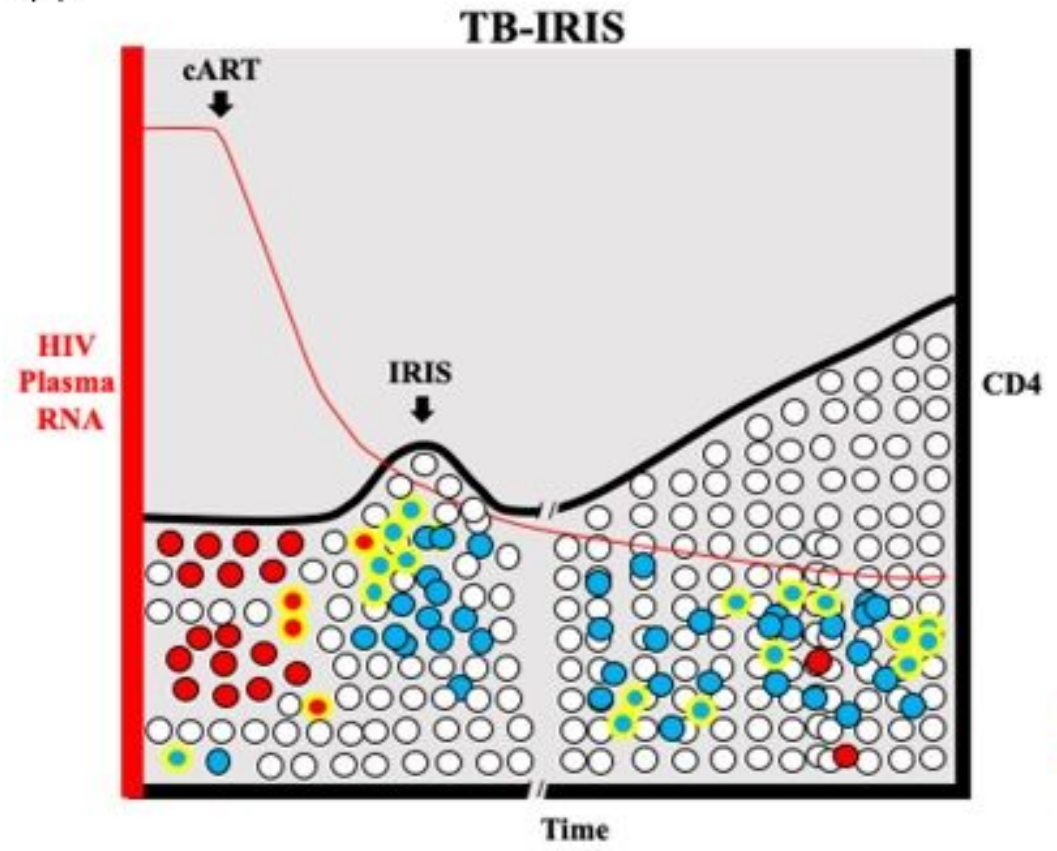

HIV infected cells

- HIV infected, TB-specific cells

Uninfected cells

\section{Figure 6}

Fundamental shifts in HIV populations with IRIS. (a) With active TB and no IRIS, there is a genetically diverse HIV infected cell reservoir that persists with cART and undergoes clonal expansion. The HIV reservoir is dominated by infected cell lineages responsible for virus production at the time of cART initiation. HIV infected cell populations include TB-specific cells, which remain part of the viral reservoir after TB resolves. (b) TB-IRIS shifts the composition of HIV infected cell populations that persist on cART. The HIV reservoir is no longer dominated by cell lineages responsible for virus production at the time of CART initiation, but rather, by distinct populations of cells. TB-specific HIV infected cells are present in the 
presence or absence of IRIS and persist for prolonged periods likely by both antigen induced clonal expansion and homeostatic proliferation.

\section{Supplementary Files}

This is a list of supplementary files associated with this preprint. Click to download.

- SupplementaryFiguresTables.docx 